\title{
Theory of Operation for the Ball Rheometer
}

\author{
C. L. Shepard \\ J. B. Colson \\ K. O. Pasamehmetoglu \\ C. Unal \\ J. N. Edwards \\ J. Abbott
}

December 1994

Prepared for the U.S. Department of Energy under Contract DE-AC06-76RLO 1830

Pacific Northwest Laboratory

Operated for the U.S. Department of Energy

by Battelle Memorial Institute

\% Battelle 


\title{
DISCLAIMER
}

This report was prepared as an account of work sponsored by an agency of the United States Government. Neither the United States Government nor any agency thereof, nor Battelle Memorial Institute, nor any of their employees, makes any warranty, expressed or implied, or assumes any legal liability or responsibility for the accuracy, completeness, or usefulness of any information, apparatus, product, or process disclosed, or represents that its use would not infringe privately owned rights. Reference herein to any specific commercial product, process, or service by trade name, trademark, manufacturer, or otherwise does not necessarily constitute or imply its endorsement, recommendation, or favoring by the United States Government or any agency thereof, or Battelle Memorial Institute. The views and opinions of authors expressed herein do not necessarily state or reflect those of the United States Government or any agency thereof.

\author{
PACIFIC NORTHWEST LABORATORY \\ operated by \\ BATTELLE MEMORIAL INSTITUTE \\ for the \\ UNITED STATES DEPARTMENT OF ENERGY \\ under Contract DE-ACO6-76RLO 1830
}

Printed in the United States of America

Available to DOE and DOE contractors from the

Office of Scientific and Technical Information, P.O. Box 62, Oak Ridge, TN 37831; prices available from (615) 576-8401. FTS 626-8401.

Available to the public from the National Technical Information Service, U.S. Department of Commerce, 5285 Port Royal Rd., Springfield, VA 22161. 


\section{DISCLAIMER}

Portions of this document may be illegible in electronic image products. Images are produced from the best available original document. 


\author{
C. L. Shepard \\ J. B. Colson \\ K. O. Pasamehmetoglu ${ }^{1}$ \\ C. Unal ${ }^{1}$ \\ J. N. Edwards ${ }^{1}$ \\ J. Abbott ${ }^{1}$
}

December 1994

Prepared for the U.S. Department of Energy under Contract DE-AC06-76RLO 1830

Pacific Northwest Laboratory Richland, Washington 99352

${ }^{1}$ Los Alamos National Laboratory Los Alamos, New Mexico 87545 


\section{Summary}

Knowledge of the rheological properties of the material contained in Hanford Site high-level radioactive waste tanks is crucial to the success of many of the mitigation and remediation methods and to the understanding of the behavior of the wastes. The ball rheometer is being developed to provide in-situ measurements of the rheological properties of the waste contained in Tank 101-SY. The need for these data arise from several sources, including modelers of tank behavior, tank safety analysts, and structural analysts. In addition, there is a need to assess the effectiveness of mitigation methods, such as the mixer pump.

Past rheological data obtained from core samples of the waste in 101-SY is suspect insofar as its reliability is concerned, owing mostly to the inability of cone-and-plate viscometers to analyze samples containing particles of sizes larger than or comparable to the gap distance between the cone and plate. It is believed that the ball rheometer can avoid many of the difficulties associated with past measurement procedures because it operates in situ. The ball rheometer measures no fluid properties directly, but rather measures drag force on the ball and the velocity of the ball. These measurements must be related to fluid properties such as the yield stress and viscosity through non-Newtonian fluid theory. A suitable fluid model for the waste is necessary in order to reduce the ball rheometer data to obtain rheological parameters.

In this report we present the ball rheometer concept and discuss the theory behind its application. Several fluid models are described, and the relationships between the drag force and velocity of the ball to rheological parameters are given for each model. These models are all steady-state models where the flow about the ball is fully developed. We also present an operational procedure for the performance of tests in the tank and give details of the components making up the ball rheometer system. Descriptions of testing in the convective or mixed layers and in the nonconvective layer are described. In addition, an error analysis is presented showing which measurements are most important in determining the accuracy of derived rheological parameters. This analysis was based on the assumption that a Bingham fluid model is representative of the waste. The error analysis shows that the performance of the ball rheometer depends on the actual values for the yield stress and viscosity in addition to measurement errors. Relatively low viscosities (less than $10,000 \mathrm{cP}$ will be difficult to measure with accuracy, especially if the fluid has a high yield stress (greater than 3500 $\mathrm{Pa}$ ). A high yield stress will also require that the ball be manually assisted to the tank bottom, rather than proceeding there under gravity.

The ball viscometer is expected to have different capabilities in different layers of the fluid. In the convective or mixed layer, which is characterized by a near zero yield stress and a relatively low viscosity, the ball rheometer is expected to be able to determine fluid density quite accurately. Only an upper bound to the viscosity is expected. It should also be able to distinguish the locations where fluid boundaries exist, such as between the convective layer and the nonconvective layer. In the nonconvective layer where the yield stress and viscosity are expected to be high enough, the ball rheometer should be able to measure both yield stress and viscosity to within about $50 \%$ of true values, but may have difficulty in accurate measurements of density. We feel that the largest obstacles in development of a successful ball rheometer are in the determination of a reasonably accurate fluid model for the waste and the nature of the waste, including waste inhomogeneities. 
The ball rheometer is expected to provide the following information:

1. fluid density to $5 \%$ of true value in the convective or mixed region,

2. yield stress and viscosity to $50 \%$ of true value in the nonconvective layer,

3. location of fluid interfaces where fluid properties change abruptly, and

4. data which allow a determination of the effectiveness of mitigation methods, such as the mixer pump.

The ball rheometer is expected to provide interpretable data when the measurement parameters are within the following ranges:

1. fluid density is greater than $1 \mathrm{gm} / \mathrm{cm}^{2}$ and the fluid has no significant yield stress

2. viscosity is greater than $10,000 \mathrm{cP}$, and

3. yield stress is less than 70,000 dynes $/ \mathrm{cm}^{2}(1 \mathrm{psi})$.

The degree to which these measurement objectives are accomplished will form the basis for measuring the success of the ball rheometer. 


\section{Contents}

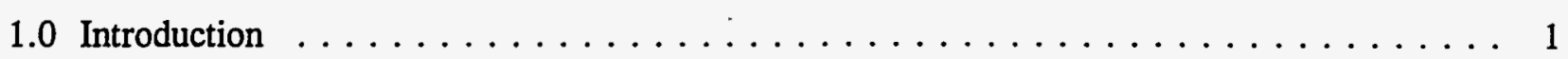

2.0 Past Measurements with Simulated Waste $\ldots \ldots \ldots \ldots \ldots \ldots$

3.0 In-situ Ball Rheometer Measurements in Tank 101-SY . . . . . . . . . . . . . . 12

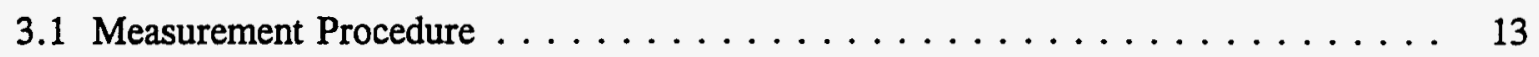

3.1.1 Case I. Unmixed Liquid Layer Over Undisturbed NC Layer . . . . . . 13

3.1.2 Case II. Mixed Layer Above Unmixed Sludge Layer . . . . . . . . . . 17

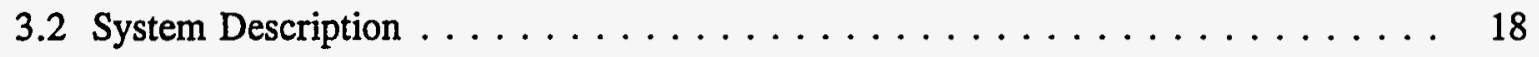

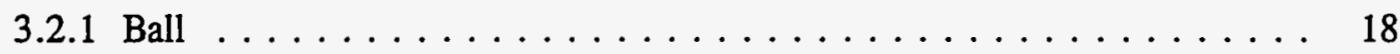

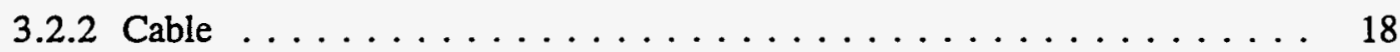

3.2 .3 Load Cells . . . . . . . . . . . . . . . . 18

3.2.4 Counterbalance Weight $\ldots \ldots \ldots \ldots \ldots \ldots$

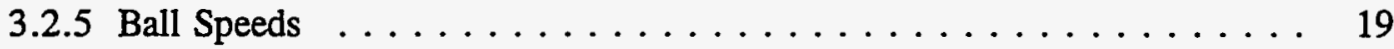

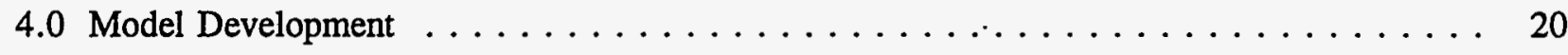

4.1 Data Reduction Methodology . . . . . . . . . . . . . . . . 21

4.1 .1 Newtonian Fluids . . . . . . . . . . . . . . . . . . 22

4.1.2 Bingham Plastics and Generalized Bingham Plastics ......... 23

4.1 .3 Power Law Fluids $\ldots \ldots \ldots \ldots \ldots \ldots \ldots$

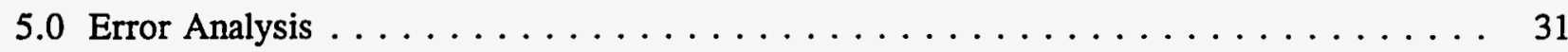

6.0 Additional Laboratory Measurements $\ldots \ldots \ldots \ldots \ldots \ldots \ldots \ldots \ldots$

6.1 Tests to Establish Expected Ball Rheometer Behavior $\ldots \ldots \ldots \ldots \ldots$. . . . 41

6.2 Model Development Tests $\ldots \ldots \ldots \ldots \ldots \ldots \ldots \ldots \ldots \ldots \ldots \ldots$

6.2.1 Waste Simulant Characterization Tests $\ldots \ldots \ldots \ldots \ldots$. . . . 42 
6.2.2 Falling-Ball Tests with Yielded Waste Simulant . . . . . . . . . . 42

6.2.3 Falling-Ball Tests with Unyielded Waste Simulant . . . . . . . . . 42

6.2.4 Viscosity Measurements with Other Methods, Using the Yielded Waste

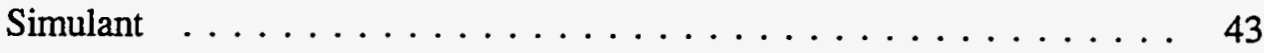

6.2.5 Flow Visualization in Falling-Ball Tests to Determine the Shape of Yielded Region $\ldots \ldots \ldots \ldots \ldots \ldots \ldots \ldots \ldots \ldots \ldots$

6.2.6 Falling-Ball Tests with 3.6-in. Diameter Tungsten Ball . . . . . . . . 43

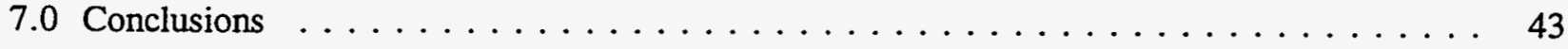

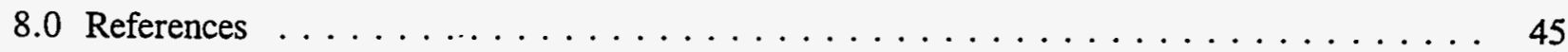




\section{Figures}

1. Apparent Viscosities Measured by Three Different Methods for a Non-Newtonian

Potassium Polyacrylate-Water Mixture $\ldots \ldots \ldots \ldots \ldots \ldots \ldots$

2. Apparent Viscosities Measured by Three Different Methods for a Non-Newtonian

Bentonite-Water Polyacrylate-Water Mixture Involving Suspended Fine Particles $\ldots . . .8$

3. Apparent Viscosities Measured by Three Different Methods for a Non-Newtonian Waste

Simulant Involving Suspended Crystalline Particles $\ldots \ldots \ldots \ldots \ldots \ldots \ldots$

4. Apparent Viscosities Measured by Three Different Methods for a Non-Newtonian Waste

1-Day-Old Waste Simulant Involving Suspended Crystalline Particles $\ldots \ldots \ldots \ldots$

5. Comparison of Viscosity of Simulated Waste Using a Cylindrical Spindle and Falling Ball . . 11

6. Diagram of In-Tank Apparatus $\ldots \ldots \ldots \ldots \ldots \ldots \ldots \ldots \ldots \ldots$

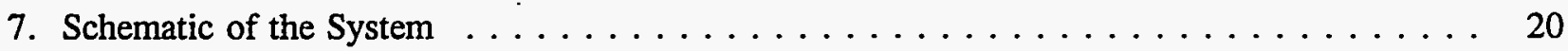

8. Drag Force on a Sphere with a Diameter of $0.1016 \mathrm{~m}$ in a Newtonian Fluid for Various

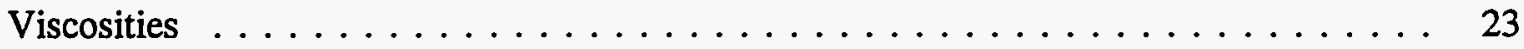

9. Calculated Drag Force (T) as a Function of Yield Stress $\left(\tau_{0}\right)$ and Viscosity $\left(\mu_{0}\right)$ for a Ball

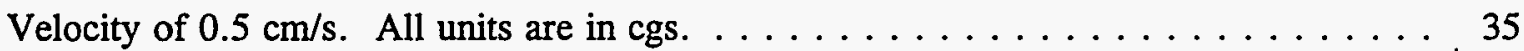

10. Calculated Drag Force $(T)$ as a Function of Yield Stress $\left(\tau_{0}\right)$ and Viscosity $\left(\mu_{0}\right)$ for a Ball

Velocity of $1.0 \mathrm{~cm} / \mathrm{s}$. All units are in cgs. . . . . . . . . . 35

11. Fractional Error in Derived Viscosity for a Range of Viscosities and Yield Stresses. A

Bingham plastic fluid model is assumed. $\ldots \ldots \ldots \ldots \ldots \ldots$

12. Drag Force Measured in a Chemical Simulant Using a 2.5-cm-Diameter Ball with a

Velocity of $0.7 \mathrm{~cm} / \mathrm{s}$. A steady-state flow is apparently obtained . . . . . . . . 39 
13. Drag Force Measured in a Chemical Simulant Using a 2.5-cm-Diameter Ball with a

Velocity of $1.0 \mathrm{~cm} / \mathrm{s}$. No steady-state region is apparent $\ldots \ldots \ldots \ldots \ldots$

14. Drag Force Measured in the Same Chemical Simulant on Different Days . . . . . . . 40

\section{Table}

1. Calculated Values for Drag Forces $\left(T_{1}\right.$ and $\left.T_{2}\right)$ for Specific Values of Velocity (V),

Yield Stress $\left(\tau_{0}\right)$, Viscosity $\left(\mu_{0}\right)$, and Ball Radius $(\mathrm{R}) \ldots \ldots \ldots \ldots \ldots$ 


\subsection{Introduction}

The Hanford Site contains over 100 high-volume tanks containing high-level radioactive wastes. The tank which has received the most attention is Tank 101-SY, which is a double shell tank containing a caustic mixed-waste slurry. The ball rheometer developed in this work is intended at least initially for application in this tank. Tank 101-SY is known to periodically release flammable and toxic gases during events known as rollovers. The tank waste is largely made up of two layers, a supernatant liquid layer underneath which is a thick sludge layer. The two layers are called the convective (C) and the nonconvective (NC) regions, so called because of the thermal transport properties ascribed to each. Although they have significant uncertainty, the current rheology data suggest the existence of a yield stress in the highly viscous nonconvective layer. Gas generated in the waste can be held in the NC layer if the material yield strength or its viscosity is high enough. Gas cannot be held in the C layer to any appreciable extent unless it is in solution. As gas continues to be generated by chemical or other processes, the number of gas bubbles and/or their sizes increases in the NC layer. A rollover occurs when the amount of gas trapped in the nonconvective region becomes great enough to overcome forces holding it in place. These forces are believed to be dependent on the rheology of the nonconvective region and perhaps the bubble surface tension. The buoyancy forces on the bubbles exceed the restraining forces arising from the yield stress and the viscosity of the NC layer. Rheology is then seen to be quite important in determining the nature of gas release events in this tank. A review and evaluation of rheological measurement methods applicable to Tank 101-SY is given in a report by Pasamehmetoglu et al. (1993). Part of the reason for development of the ball rheometer is to determine the yield strength and apparent viscosity of the NC layer so that modeling of tank behavior through codes such as TEMPEST can become more reliable. However, characterization of the rheological properties of this layer is not desired solely for modeling purposes. Other groups involved with the tank safety and structural analysis are also interested in the rheological parameters of the NC layer.

In addition, there is keen interest in determining the mitigation effectiveness of mixer pumps installed in Tank 101-SY. The mixer pump mixes the NC layer with the $C$ layer by directing high-speed liquid streams into the NC layer, thereby diluting and displacing the sludge material. The working fluid is liquid from the $C$ layer. Presumably a mixed layer is created which has a very low yield stress and the volume of the NC layer is diminished. The gas retention capability of the waste is therefore reduced. In this instance, attention will be given primarily to the density, rheology, and spatial extent of the region where waste from the nonconvective layer has been mixed with the waste of the convective region. Details on the requirements of users of the rheological data which will be gathered by the ball rheometer are given in a report by Shepard (1993).

Some of the conditions within the tank are known from in-situ instrument arrays and from past tank sampling. Most of the rheological information obtained to date comes from hot cell testing of core samples drawn from Tank 101-SY.,2 We will discuss these data shortly. Roughly, there is a

'Allemann, R. T. June 3, 1992. "Viscosity and Shear Strength of 241-SY-101 Slurry," Pacific Northwest Laboratory memorandum to $\mathrm{M}$. Kreiter. 
liquid layer of about $15 \mathrm{ft}$. in depth and beneath that is the nonconvective layer, also of about $15 \mathrm{ft}$. depth. The unmixed liquid layer is very nearly Newtonian in behavior with a fairly low viscosity (about $25 \mathrm{cP}$ ). This layer is of minor interest in the unmixed form. The NC layer has a high particle concentration and possesses a yield strength as well as a high viscosity after yielding. The temperature of the waste is around $45^{\circ} \mathrm{C}$ and exhibits a time and spatially dependent variation in the NC layer. Mixing by convection is believed to be why the liquid layer shows little spatial thermal variation. The densities of the $\mathrm{NC}$ and $\mathrm{C}$ layers are not too different, with specific gravities of about 1.7 and 1.5, respectively. The surface level of the tank varies between cyclic gas release events, and this is due to generation and buildup of gas within the NC layer. Variations of the order of $1 \mathrm{ft}$. have been observed in the level of the surface.

Past measurements of the rheological properties of the waste in Tank 101-SY have shown some anomalous behavior and there is some question as to the reliability of these data. Rheology measurements have been made on samples obtained from the tanks and placed in a hot cell. For example, core samples from Tank 101-SY were taken during windows $\mathrm{C}$ and $\mathrm{E}$; those samples were used for rheology measurements. ${ }^{2}$

These measurements show that there is considerable difference in the convective layer viscosity for samples from windows $\mathrm{C}$ and $\mathrm{E}$. While window $\mathrm{C}$ samples show that the convective layer is nearly Newtonian, with a viscosity on the order of $10 \mathrm{cP}$, the window E samples show a highly nonNewtonian behavior, with very high viscosities (on the order of $10^{6} \mathrm{cP}$ ) at low shear rates, even for the convective layer. The core samples were obtained during a window (within 2 to 3 weeks) following a GRE while the waste was in its settling stage. Thus, one speculation is that the sample obtained during window E actually corresponded to a chunk of a nonconvective (NC) layer while this chunk was settling down through the convective layer. There are other possible explanations for these anomalous observations, most of which have to do with the procedures used for sample capture and sample handling.

Interpretation of rheology measurements for waste from the NC layer also present some challenges. Typically, the rheograms show that the shear stress is a decreasing function of the strain rate at low strain rates. This behavior is not observed as a quasi-steady and stable rheogram for any other material (and/or slurries) investigated in the literature. Besides measurement errors, this behavior can only be explained by the "disordered to ordered" transition in highly concentrated suspensions (Merrington and Woodcock 1991). However, such transition is highly transient and metastable in nature and should not be used as a quasi-steady rheogram. Later attempts were made to quantify the thixotropic behavior of the NC layer samples by running experiments at constant shear rate for several minutes and recording the shear stress. From the behavior of the curve, we concluded that several minutes was the time constant of the material, and the waste sample had reached equilibrium in those tests. ${ }^{3}$ However, when plotted on a stress-strain rate curve, the results were similar to the transient rheograms because there was a region where the stress was a decreasing function of a strain rate. It is worth mentioning that, according to Merrington and Woodcock (1991), the transition from

\footnotetext{
${ }^{2}$ Tingey, J. M. May 1992. "Rheological Properties of Waste from Tank 101-SY," Pacific Northwest Laboratory draft report.

${ }^{3}$ Hall, M. N. and J. R. Phillips. August 21, 1992. "Thixotropic Viscosity Issue--10 min. SA Limit," Westinghouse Hanford Company memorandum to J. W. Lentsch.
} 
a disordered to ordered arrangement in highly concentrated suspensions can be a very slow process and may take "many hours, possibly days." If one uses the transient rheogram in quantifying the quasi-steady behavior of a system, unrealistic results and conclusions follow. For example, if we were to use the current rheograms to design a waste retrieval system consisting of a retrieval pump and piping system, in a certain range of parameters, we would conclude that increasing the pump head would result in a reduction in flow.

The temperature dependence of the NC layer viscosity also requires further investigation. The data indicate that over a certain range of temperatures and strain rates, viscosity increases with increasing temperature, in marked contrast from most fluids. Currently, we overlook this empirical behavior and model the viscosity as being a decreasing function of temperature similar to pure liquids. ${ }^{1}$ However, the observed behavior has been reported in the literature for suspensions and may be real (see, for instance, Fig. 6.15 in Ferguson and Kemblowski 1991). This observation has important consequences if one were to use heating as a mitigation or remediation scheme.

Finally, the experimental methods used to establish the rheology of the NC waste layer also has a number of drawbacks. The disturbance caused during removal and storage of the samples is likely to influence the rheological properties. Likewise, the viscometers (cone and plate) used in those measurements require small sample sizes. The placement of these small amounts of material into the viscometer is likely to create additional changes in the rheological properties. Also, the particles in the waste slurry are believed to be 1 to $100 \mu \mathrm{m}$ in size. For a cone-and-plate viscometer used in the hot-cell measurements, this particle size is likely to interfere with the gap, resulting in erroneous viscosity or yield stress measurements. At Los Alamos National Laboratory this effect was investigated using a simulated waste containing salt crystals with similar sizes to the salt crystals in the real waste (Piccola et al. 1993). The results indicate that while repeatable data with small scatter could be obtained using spindles, the cone-and-plate data are unrepeatable and exhibit erratic scatter. The postulated mechanism for this behavior was that the narrow gap in the cone-and-plate viscometer (13 $\mu \mathrm{m})$ resulted in grinding up the salt crystals and was not capable of quantifying the viscoplastic behavior of the mixture. In most cases, the viscosity measured using the cone-and-plate viscometer was an order of magnitude greater than the viscosity indicated by the spindles. We summarize the above mentioned study and its findings briefly in the next section.

There are direct and indirect methods for laboratory measurements of yield stress (see Dzuy and Boger 1983). Indirect methods are based on the interpretation of fundamental shear-stress shear-rate data. The yield stress is obtained by extrapolating the shear-stress shear-rate curve as the shear rate goes to zero. In practice, this straightforward method is not usually possible with the use of commercially available viscometers because the low shear rate data are not available. Even when low shear rate data are available, the instrumentation uncertainty and other errors due to slip flow make this method questionable (Goodrich et al. 1989). In addition, this method requires that the rheogram be identified correctly. The use of a different fluid model also results in different yield stress quantities. The direct methods involve the vane method (Dzuy and Boger 1985, Keentok et al. 1985), stress relaxation method, and measuring the shear stress at which flow first begins under constant shear stress, or shear rate conditions. There are other special methods, such as a cylindrical specimen between two parallel plates under constant load (Navickis and Bagley 1983); penetration of solid objects, such as cone and plate, needle, or sphere into an initially horizontal test specimen; application of constant torsional moment to one end of a rod or hollow cylinder made of the test material; or measuring the drag force acting on a stationary plate submerged in a moving test material (see Dzuy and Boger 1985). Some of these methods are not applicable to fluid materials and the success of 
these techniques depends on the capability of the test material to exhibit well-defined yielding under selected experimental conditions. However, there are still questions that the quantity obtained by these tests can be identified as true yield stress. Usually, the yield stress is measured by several methods for comparison purposes.

The yield stress of 101-SY waste samples was measured by the vane method, which is the method of measuring the shear stress at which flow first begins under constant shear-stress or shear-rate conditions. $^{2}$ There are significant differences (about $100 \%$ ) between quantities obtained from the vane method and from the method of measuring the shear stress at the onset of flow under constant shear rate. It is worth noting that when the quantities obtained from the two methods mentioned above are compared with the quantity obtained from the indirect method (extrapolation of shear stress to zero shear rate), the yield stresses differ by orders of magnitude.

Currently, we do not have any data to correlate the effect of dilution on the waste rheology. Person ${ }^{4}$ measured the viscosity of a mixture of the $\mathrm{C}$ and $\mathrm{NC}$ layers, using the proportions that are believed to exist in the tank. Roughly, the mixture consists of $100 \%$ dilution of the NC layer with the $\mathrm{C}$ layer. The mixture exhibited a shear thinning behavior with viscosities ranging from $\approx 400$ (low shear rate) to $50 \mathrm{cP}$ (high shear rate) over the range of 5 to $1000 \mathrm{~s}^{-1}$ shear rates. The measurements were done at room temperature using a cylindrical bob spinning inside a fixed outer cylinder (spindle). These spindle measurements provide one data point for the dilution effects. However, we believe further analysis of the data is required to confirm that the mixture remains homogeneous during the spinning action and that migration of the solids as a result of centrifugal forces does not affect the measurements.

The above discussion points out the need for additional rheology data on the waste in Tank 101-SY. Some measurements have simply not been made while others are suspect primarily because of the difficulty of obtaining measurements with the waste using standard laboratory measurements. As evidenced in a report outlining the needs of the data users community (Shepard 1993), it is important to have knowledge of the waste's rheological properties to sufficient precision. so that the behavior of the waste can be understood from numerical modeling and so that safety and structural concerns can be adequately addressed. In addition, the effectiveness of mitigation strategies, such as the mixer pump, can be measured with rheology data.

The ball rheometer, which is the subject of this paper, is proposed as at least a partial solution to these data needs. The intent of ball rheometry is to provide further rheological information (shear stress at low shear rates, apparent viscosity at low shear rates, and density) from in-situ measurements of 101-SY waste. It is believed that it will be most successful at measurements of the yield stress and zero shear rate viscosity of the NC layer and the density of the $\mathrm{C}$ layer or a mixed layer. It should also be very effective at determination of the boundaries between the NC layer and the $\mathrm{C}$ or mixed layer. None of the quantities mentioned above are directly measured with the ball rheometer. Rather, these quantities must be inferred through theoretical relationships connecting measured quantities to rheological properties. A reasonably accurate model for the fluid is necessary, particularly for the NC layer and the mixed layer. Laboratory studies using chemical simulants of the waste will be relied upon to help develop the model, as will the actual data collected in Tank 101-SY.

${ }^{4}$ Person, J. C. September 2, 1992. "Gas Retention Tests on 101-SY Tank Waste After Mixing." Westinghouse Hanford Company internal memorandum to J. W. Lentsch. 
Fortunately, there is no identified requirement for very precise determination of rheological parameters. It is sufficient to determine viscosity and yield stress to within about $50 \%$ of true value in the $\mathrm{NC}$ layer, while density information is most important in the $\mathrm{C}$ layer or mixed layer where errors of several percent are acceptable.

There are circumstances which can result in partial failure of the ball rheometer, most notably in the NC layer. Our inability to guarantee success here results mostly from an incomplete knowledge of this layer. If the layer is highly stratified or if the layer is inhomogeneous to a sufficient extent, we will have difficulty obtaining the rheological parameters to sufficient precision. We will be able to determine that it is stratified or inhomogeneous, however. The requirement for homogeneity at least over some limited depth of the waste is due to theoretical limitations wherein rheological properties are related to measured quantities only under the conditions of steady-state fluid flow. The theoretical description of transient phenomena has not been suitably addressed to date.

The measures by which ball rheometry will be considered successful are listed below.

1. If a reasonably accurate fluid model describing the NC layer can be developed and if yield stress and zero shear rate viscosity can be determined to within $50 \%$ of true value from this model and the actual measurements, ball rheometry has served one of its purposes.

2. If the density of the $C$ layer or mixed layer can be determined to within $5 \%$, the ball rheometer has provided meaningful results. It is assumed here that the mixed layer will have no significant yield stress.

3. If the interface between different liquids where rheological properties abruptly change, such as between the $\mathrm{C}$ or mixed layer and the NC layer, can be determined to within $1 \mathrm{ft}$, the ball rheometer is successful. It should be remembered that this boundary may be somewhat diffuse if the $C$ layer is replaced with a mixed layer.

4. If the ball rheometer can provide information as to the effectiveness of various mitigation strategies, such as the mixer pump or perhaps the sonic probe, then it is successful. Viewed in this sense, the ball rheometer would need to possess the ability to measure changes in the fluid properties.

It is presently believed that the ball rheometer will be capable of meeting the above success criteria.

The remainder of this report is organized as follows. In Section 2 we present the results of past testing with simulated waste. In Section 3 we show present plans for performing testing in the tank, including a description of the ball rheometer system. A discussion of various fluid models which may be applicable to this problem is given in Section 4. A discussion of errors and their propagation is presented in Section 5. The need for further laboratory experiments and a description of these tests make up Section 6, and Section 7 is a summary.

This report is not the final word on the topics covered here. A subsequent report will follow which outlines the final test procedure to be followed in experiments performed in Tank 101-SY. This report will also contain an analysis of errors based on the best available fluid model and finalized hardware specifications. The results of the experimental tests described in Section 6 will also be presented. 


\subsection{Past Measurements with Simulated Waste}

In order to understand the reasons why ball rheometry is being used as the method of choice for insitu measurement of rheological properties, we should begin by reviewing experimental results obtained using chemical simulants. As suggested in the previous section, standard rheological measurements have not provided consistent results.

Los Alamos initiated work (Piccola et al. 1993) to evaluate the adequacy of the cone-and-plate viscometry, including difficulties associated with this measurement method and repeatability of tests for the waste materials. There was a lack of repeatability in tests reported. ${ }^{2}$ Also, Hall and Phillips ${ }^{3}$ mentioned that they did not obtain reasonably similar data when a test was repeated.

To compare the cone-and-plate measurements with other methods, we selected the following additional techniques:

- cylindrical spindle,

- cylindrical spindle with a small sample adapter, and

- falling-ball viscometer (performed subsequent to the experiments of Piccola et al. 1993).

First, the spindles and the cone-and-plate techniques are based on the measurement of torque in a shear flow field. The cone-and-plate viscometer involves two plates separated (one being inclined $5^{\circ}$ ) with a small clearance $(13 \mu \mathrm{m})$. The bottom plate is rotated with a constant speed, and the torque is measured. The measured torque is converted to the shear stress at a given shear rate. Each measurement gives a steady-state point on the rheogram. We could also record the shear stress as a function of time as it reaches the steady-state conditions. The cylindrical spindle is a simple rotating cylinder in a larger cylindrical sample container. The small sample adapter technique also uses cylindrical spindles with the sample in a smaller container. The falling-ball method is based on the measurement of the terminal velocity of a ball falling through a stagnant sample. We have only preliminary data taken with this method. For a given fluid sample, we obtain the shear-stress shearrate (or viscosity-shear rate) relationship for each method. Below, we briefly summarize our findings, i.e., the comparison of rheology results we obtained from each technique.

The cone and plate and spindles were calibrated with the Newtonian calibration fluids at different temperatures. Each method gave the same results, with excellent repeatability.

When we used a non-Newtonian fluid with no particles [potassium polyacrylate-water mixture (Arigel)], the cylindrical spindle and cone and plate gave reasonably similar results, and the repeatability of tests was reasonably good, as shown in Figure 1 . The tests with the small sample adapter indicated some disagreement with values obtained from the cylindrical spindle at lower shear rates. It is worth remembering that the sample was mixed before each experiment was started and that the shear rate was increased stepwise (see Piccola et al. 1993 for details). The data at each shear rate were taken at several different intervals, involving a minimum of five revolutions. Uncertainty bars including instrumental uncertainty are shown in Figure 1 and elsewhere to indicate the range of variations of separate measurements at any given shear rate. If no uncertainty bars are presented for a data point, the size of the data point represents the instrumental uncertainty. The variation in viscosity at a given shear rate can be thought of as the measure of the change in the fluid state with 


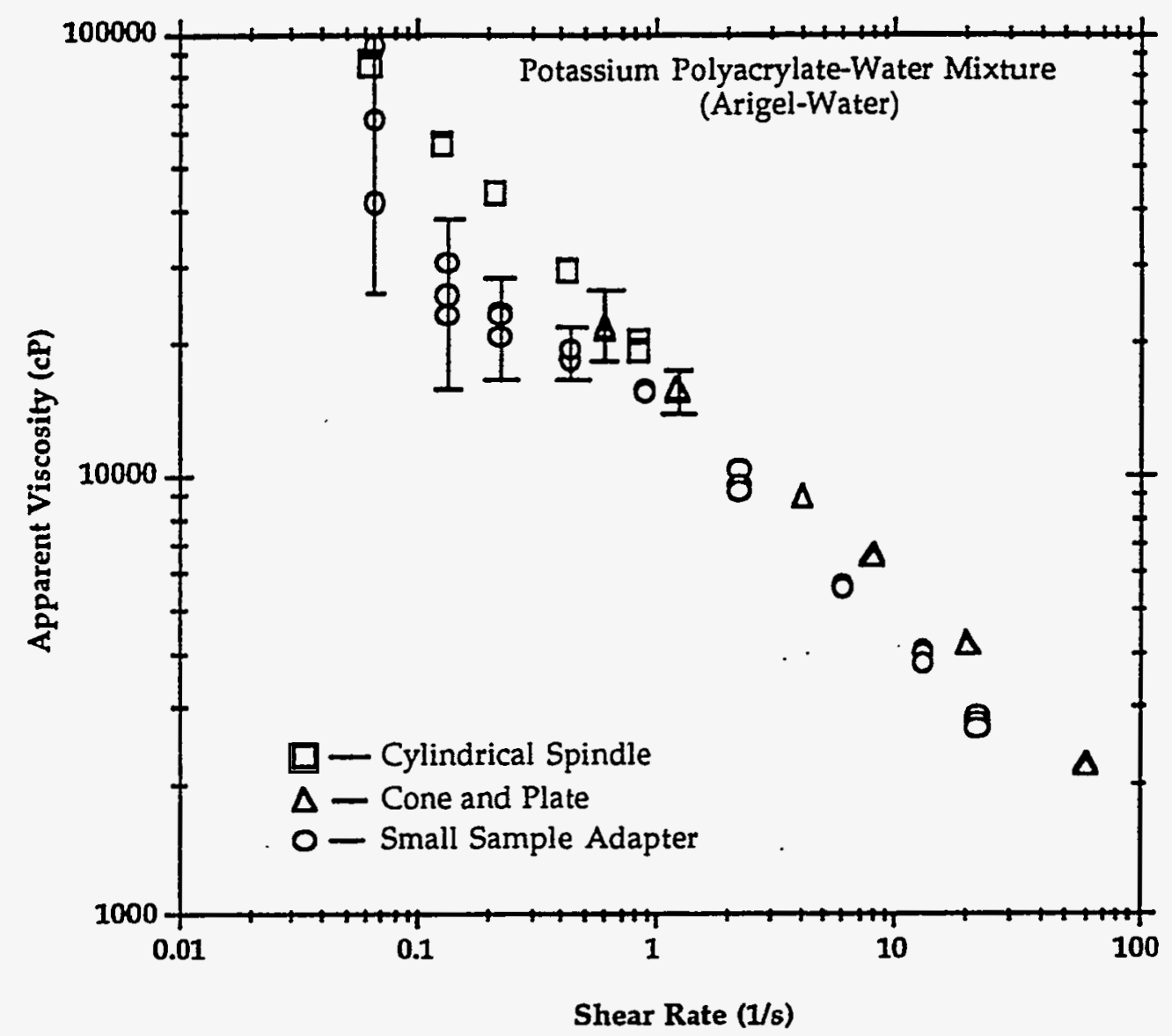

Figure 1. Apparent Viscosities Measured by Three Different Methods for a Non-Newtonian Potassium Polyacrylate-Water Mixture

time. It is evident that variation in the measured viscosity at the lowest shear rate is significant in tests with the small sample adapter. These tests give relatively lower viscosities at lower shear rates. Above a shear rate of $0.88 \mathrm{1} / \mathrm{s}$, the agreement between these three methods is reasonable.

Because waste includes crystalline particles, we also tested the first three methods with a bentonitewater mixture that included some fine particles. The bentonite-water mixture, with a volume ratio of 1 to 10 , respectively, was prepared and kept in a container for 1 day. The three methods gave reasonably repeatable and similar results for the rheology measurements, as shown in Figure 2.

Finally, we used a simulated waste that included the same chemical species as the waste in Tank 101SY. The comparison of results obtained from the cylindrical spindle, cylindrical spindle with a small sample adapter, and cone-and-plate viscometers is given in Figure 3.

The sample size necessary for the cylindrical spindle tests is relatively large $\left(500 \mathrm{~cm}^{3}\right)$. The simulated waste was mixed in the container before experiments. Small volumes taken from different locations of the container were then taken by syringe as samples of those used in the cone-and-plate device. The square symbols in Figure 3 show the apparent viscosity measured by the cylindrical spindle at the beginning of the tests. The apparent viscosity showed an exponentially decreasing trend 


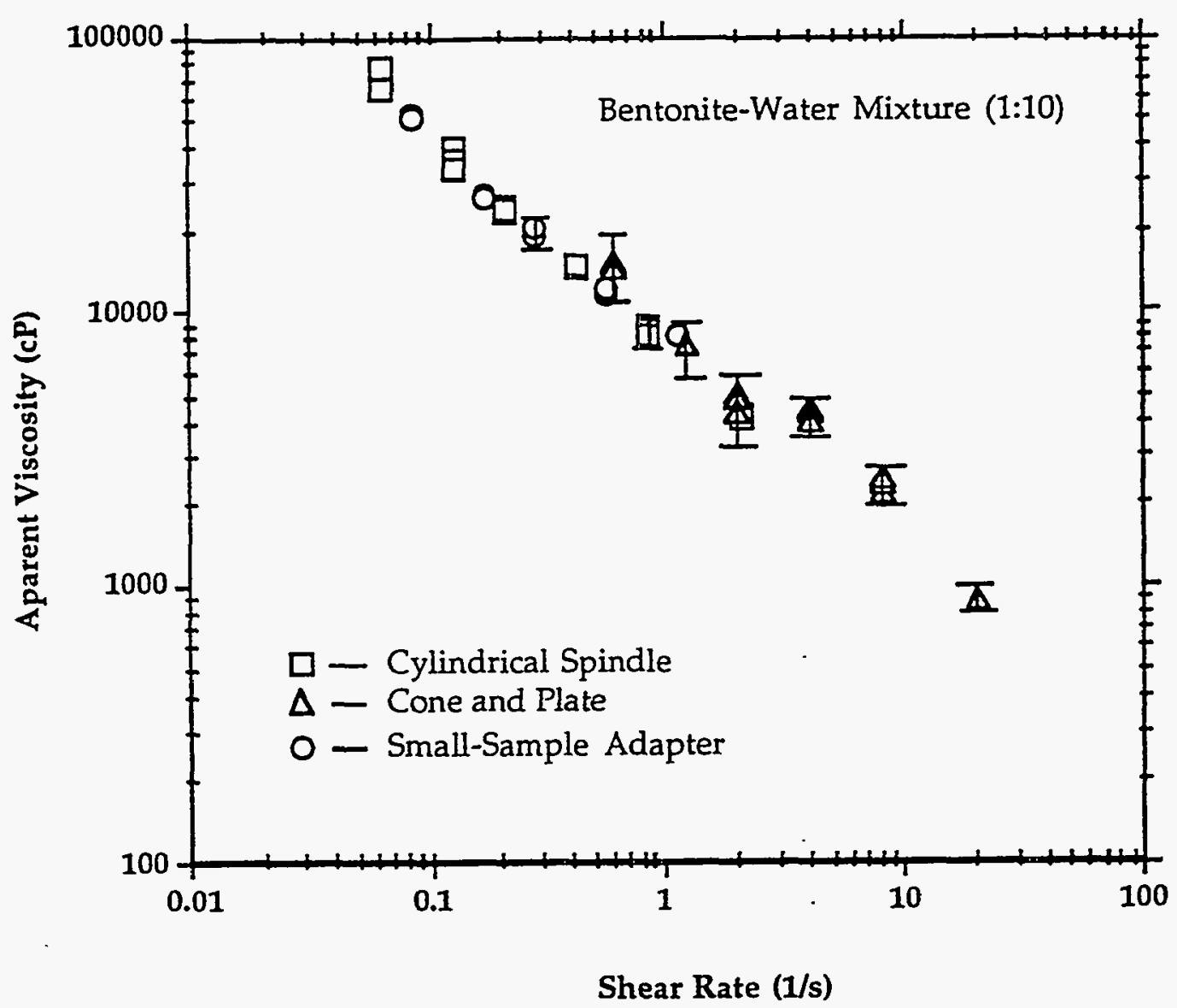

Figure 2. Apparent Viscosities Measured by Three Different Methods for a Non-Newtonian Bentonite-Water Polyacrylate-Water Mixture Involving Suspended Fine Particles

with the increasing shear rate for the cylindrical spindle tests. The variation in viscosity during a measurement at a given shear rate was negligible. However, when the sample was mixed and the cylindrical spindle tests were repeated 1 hour later, some variations, especially at lower shear rates (at the beginning of the test) were evident. The apparent viscosity tended to increase slightly in the second series of tests conducted with the cylindrical spindle. This difference shows the significance of the aging effect of the waste. Thus, the cone-and-plate measurements were conducted at the same time as the cylindrical spindle measurements to eliminate the effect of aging of the sample.

The results obtained from the cylindrical spindle with a small sample adapter are also included in Figure 3. The only difference between the cylindrical spindle and the cylindrical spindle with a small sample adapter is the gap between the container and spindle, which is relatively small with the small sample adapter. It is clear that the use of a small sample adapter results in an increase in the apparent viscosity at a given shear rate. However, the viscosity again decreases smoothly and exponentially with reasonable repeatability. Note that tests with a small sample adapter were obtained ion a different date so that simulant aging may be important. This may be one of the reasons for the shift we see in the viscosity. Another possible reason for the differences could be that particle migration may be limited in a small gap. The primary information in Figure 3 is the repeatability of a test at a given time and variation of the viscosity with the shear rate. In this sense, the use of a small sample 


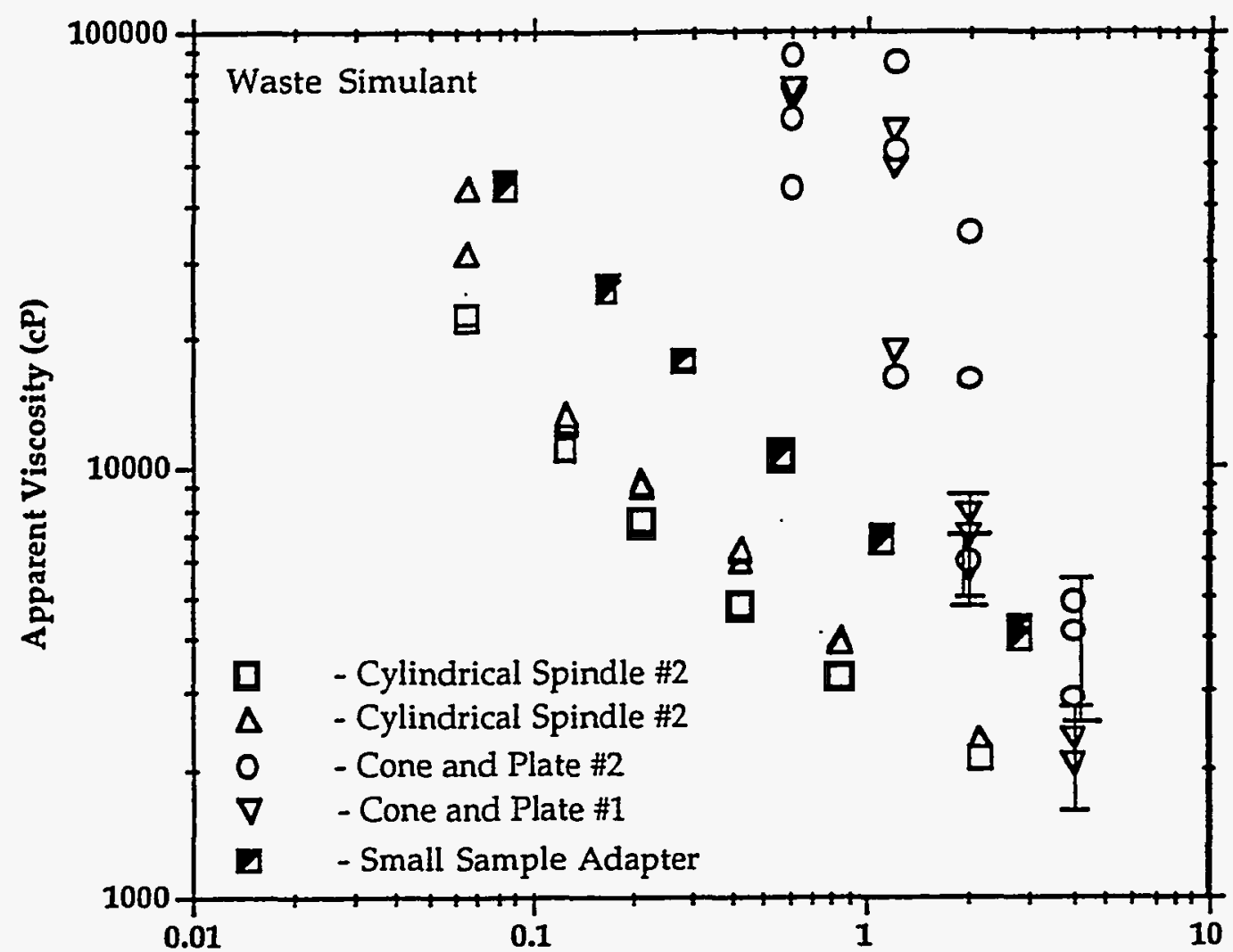

Shear Rate (1/s)

Figure 3. Apparent Viscosities Measured by Three Different Methods for a Non-Newtonian Waste Simulant Involving Suspended Crystalline Particles

adapter alșo results in reasonably repeatable results, and the measured viscosity exponentially decreases with shear rate.

The apparent viscosity at a given shear rate varied significantly during cone-and-plate tests, as shown in Figure 3. The cone-and-plate tests were performed with two different cone-and-plate sizes, which require different sample sizes (the data are represented by circles and inverse triangles). Both sizes showed significantly varying apparent viscosity during a test at a given shear rate. The repeatability of the data using the cone-and-plate device was observed to be unacceptably high. The apparent viscosity measured by the cone-and-plate device at a given shear rate is also almost an order of magnitude higher than that obtained with the cylindrical spindle viscometer.

Although we repeated the cone-and-plate tests several times on different days with different samples, we observed the lack of repeatability and measured the different viscosity each time. An example of these repeat tests is shown in Figure 4, which shows the results obtained from the 1-day-old simulant. Figure 4 indicates the same results we presented in Figure 3; the cylindrical spindle shows a reasonable variation at a given shear rate, whereas the cone-and-plate device results in relatively large variations. Also, the measured values by the cone-and-plate device are high relative to values obtained by the cylindrical spindle. 


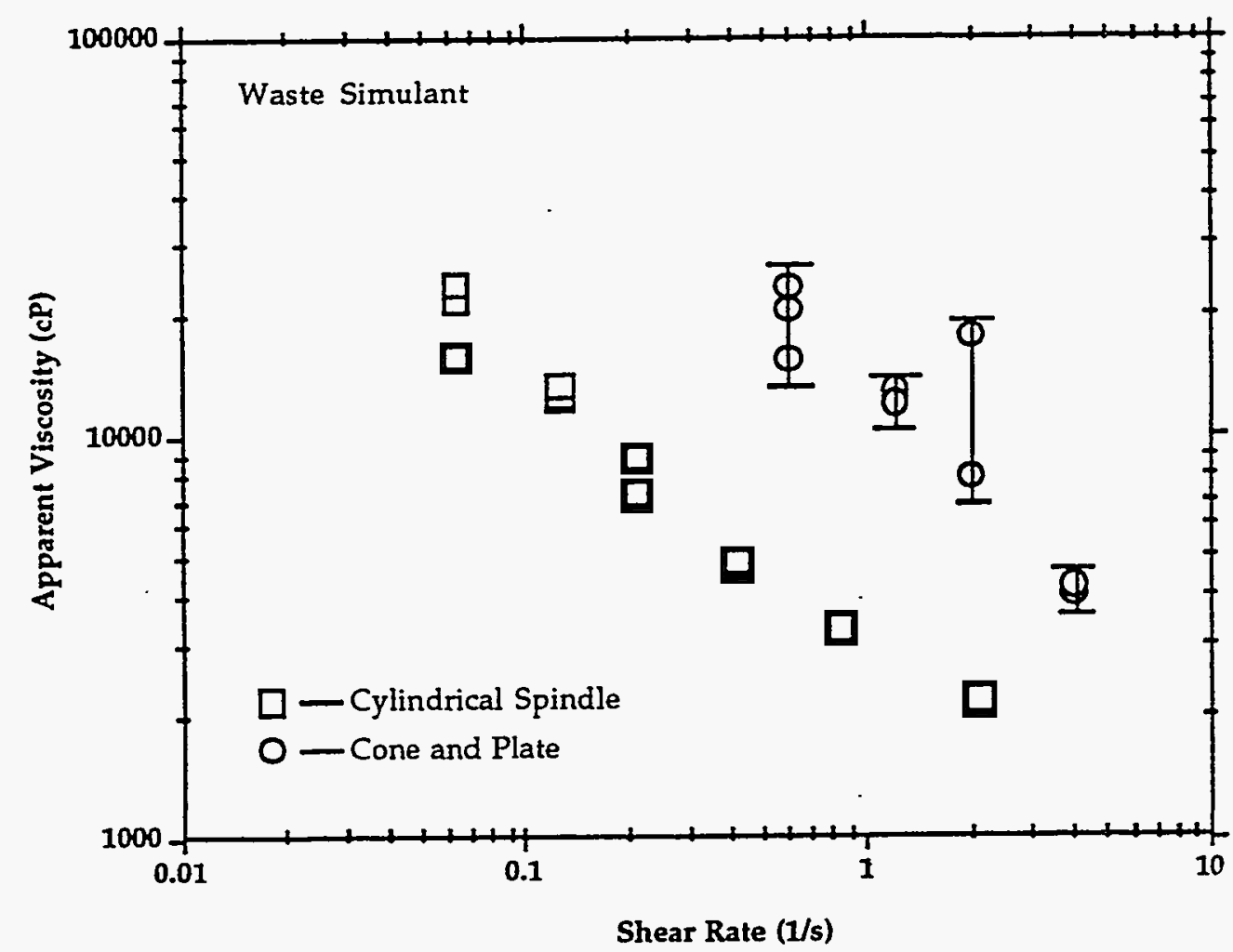

Figure 4. Apparent Viscosities Measured by Three Different Methods for a Non-Newtonian Waste 1Day-Old Waste Simulant Involving Suspended Crystalline Particles

Results shown in Figures 3 and 4 indicate that the cone-and-plate device may not yield correct measurements of the waste viscosity, and the current Tank 241-SY-101 waste rheology data could have significant uncertainty. The current waste rheology data should therefore be used with caution in any analyses.

We believe that the most important reason for the lack of repeatability is the crystalline size. The waste simulant we used included particle sizes up to $40 \mu \mathrm{m}$. Comparison of this size with the clearance of the cone-and-plate viscometer, $13 \mu \mathrm{m}$, suggests the possibility of grinding. The selection of the same particle characteristics for each cone-and-plate experiment is almost impossible when a very small sample is needed. We concluded from this study that the cone-and-plate technique is not adequate for the rheology measurements in the waste.

The data taken with cylindrical spindles show good repeatability. However, we could not conclude whether this method gives the correct measurements because there is a concern with particle migration in a shear flow. If the particles migrate, the assumption of homogenous concentration is not valid, and measurements are misleading. 
To validate the spindle measurements, we performed some preliminary falling-ball experiments at Los Alamos. The terminal velocity is determined by measuring the flight time over a given distance using $\mathrm{x}$-ray radiography (Mondy et al. 1986). Two different falling balls were used: a stainless-steel ball with a diameter of $3 / 8$ in. and a tungsten-carbide ball with a diameter of $1 / 8 \mathrm{in}$. The falling-ball viscometer gave reasonably similar viscosity results with the cylindrical spindles, as shown in Figure 5. This does not necessarily mean that both techniques give the same results because the flow fields are different and the Reynolds number may not be the correct scaling parameter. Nevertheless, it gives an idea for the first-order comparison.

The falling ball measurements showed that the fluid thinned each time a ball was dropped through it. For Newtonian fluids, viscosity is a fluid property and should not be a function of the ball size and material in this technique. This is valid when the particles are suspended homogeneously in a continuous liquid phase. Figure 5 indicates that this is not the case for this solution (tungsten-carbide and stainless-steel balls gave different results). In addition to non-Newtonian effects (where viscosity is a function of the flow field), there appears to be local disordering in the fluid structure. These are preliminary results; however, they indicate that for this particular waste simulant, the interpretation of falling ball results is also not straightforward. However, we believe that among the methods investigated, the falling ball technique is still a promising technique because the data collection occurs from undisturbed fluid as the ball falls through it. Also, it may be possible to determine an apparent viscosity to describe the waste's fluid dynamics if sufficient data are obtained using the falling ball experiments.

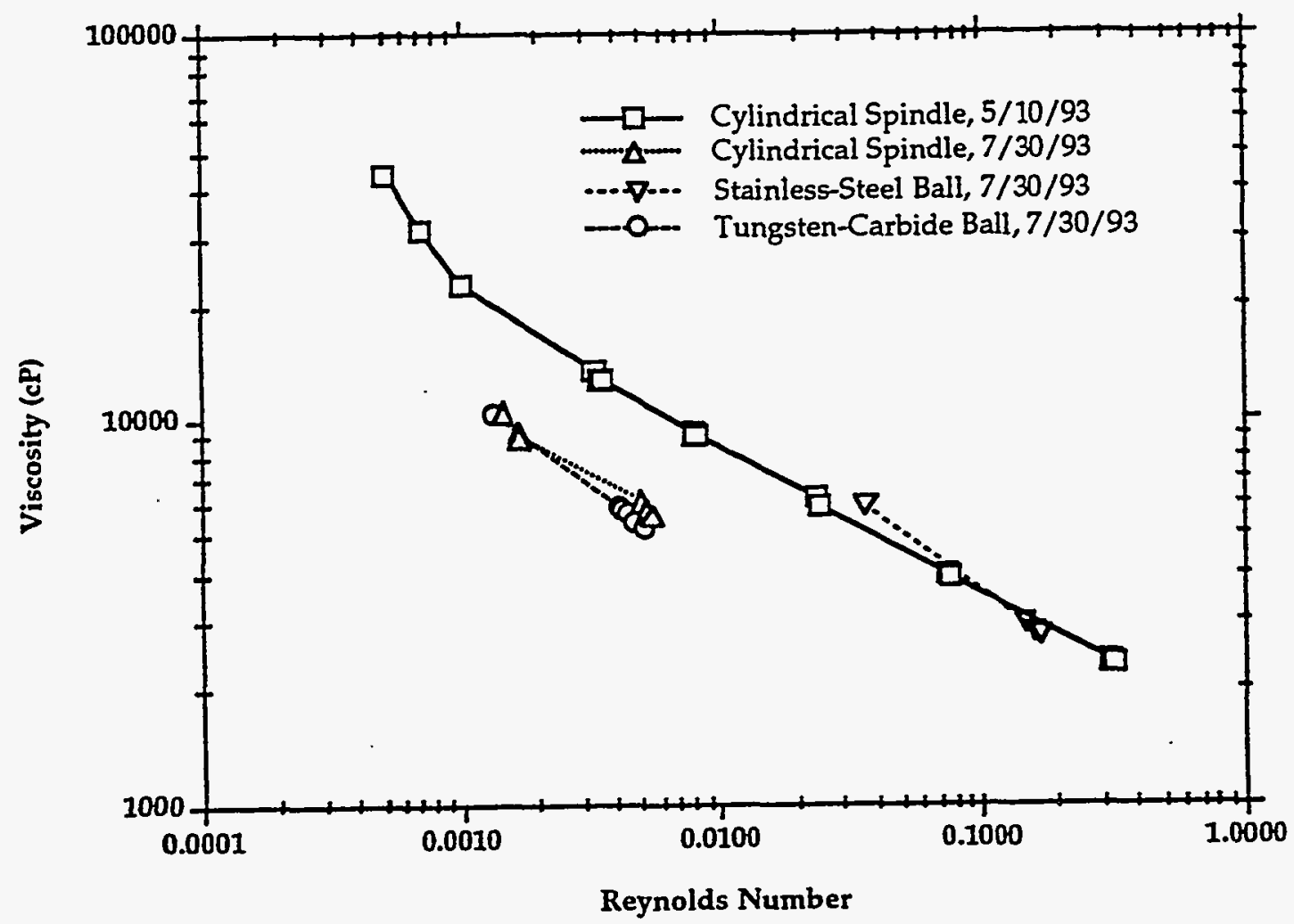

Figure 5. Comparison of Viscosity of Simulated Waste Using a Cylindrical Spindle and Falling Ball 


\subsection{In-situ Ball Rheometer Measurements in Tank 101-SY}

Accurate in-situ rheology measurements would be the best approach for determination of the character of the waste. A simple methodology for obtaining in-situ measurements is presented in this paper. However, it is important to emphasize that the ball rheometer methodology has possible shortcomings due to the range and variability of the waste material. For instance, it may provide more reliable yield stress and viscosity information in the NC layer than in the $C$ layer, and density measurements will probably be more accurate in the $\mathrm{C}$ layer than in the $\mathrm{NC}$ layer. If possible, the ball rheometer measurements should be supplemented with results obtained from hot cell testing.

The objective is to design a method that can provide in-situ measurements of the waste rheological properties. Our goal is to keep the design and operation of the equipment as simple as possible without jeopardizing the accuracy of the measurements. Naturally, there is a tradeoff between simplicity and accuracy. We believe the proposed method meets the simplicity requirements and provides a reasonable degree of accuracy (about 50\%) of rheological properties for very low shear rates. In addition, the ball rheometer minimizes the difficulties associated with deploying instrumentation within a waste tank. No electrical components or sensors will be introduced into the waste, only a ball and some cable. Therefore safety concerns, while still apparent, are considerably reduced and manageable.

One could engineer other types of in-situ measurement methods. An example recently proposed by WHC was to insert a rotational device (such as a spindle in a jacket) into the waste. This method has certain disadvantages as compared to a simpler falling-ball viscometer:

- It is more difficult to design, build, and operate.

- It disturbs the waste during insertion, whereas the disturbance and measurement can be simultaneous with a falling or raising ball.

- A falling ball makes use of the infinite waste environment, whereas trapping the local waste inside the jacket is a problem.

- It has the same problem with particle migration, as mentioned for the laboratory measurements.

- Based on the problems treated in the literature, it is easier to obtain shear strength information from falling-ball data than from spindle data.

The areas where the in-situ falling-ball viscometry has its shortcomings are common to spindle measurements. The only obvious advantage of the spindle is that one can control the shear rates. For a falling ball, the shear rates ranging from 0 to $1.5 \mathrm{~V} / \mathrm{d}$ (where $\mathrm{V}$ and $\mathrm{d}$ equal ball velocity and diameter, respectively) are integrated over the entire sheared surface, and it is necessary to use an appropriate model to reduce the data into terms of a rheogram. 
In addition, the deployment of a simple device such as a falling ball may be used to evaluate the effectiveness of various mitigation schemes that are being tested in Tank 101-SY. For instance, we can use the ball before and after pump operation in a given direction to evaluate how jet mixing changes the rheology. Also, we can use it in parts of the tank furthest away from the jet orientation to obtain data on effects such as piling of solids and local growth of the NC layer.

\subsection{Measurement Procedure}

A diagram of the device which will be used for in-situ ball theometry measurements is shown in Figure 6. The instrumentation consists primarily of a spool wound with a single layer of cable, and two pulleys around which the cable runs, and a load cell which measures twice the tension in the cable plus the weight of the pulley assembly which rides on the load cell. The cable spool is driven by an electric motor and the ball velocity is determined from an encoder attached to the spool. The encoder measures the rotational velocity of the spool from which cable velocity is determined from a knowledge of the spool diameter. The ball velocity equals the cable velocity. In almost all cases of interest, the ball will be either lowered or raised at a constant velocity while the force measured with the load cell is recorded. The acceleration of the ball from some initial velocity to some final constant velocity is somewhat adjustable. Measurements of the cable tension while the ball is at constant velocity form the most important data collected during these experiments. Ball location is easily determined from a knowledge of the ball velocity as a function of time and the reference location of the ball.. Both falling ball and raising ball experiments can be performed with this arrangement. In addition, static measurements can be performed where the ball is at rest and the apparent weight of the ball is measured with the load cell. These measurements are for determination of the waste density.

The entire assembly shown in the diagram will be housed within an enclosure and is designed to interface to a 4-in. riser pipe through which the tank waste is accessible. A decontamination system will be employed for washing the cable and ball as they are withdrawn from the tank. All motors and electronics associated with ball velocity control and measurement and load cell measurements will be housed in a weatherproof enclosure. The data will be transmitted via temporary cabling to a remote station for recording and eventual analysis.

The actual measurement procedure which will be followed has not yet been finalized. It is expected that measurements will be obtained somewhat along the following lines.

\subsubsection{Case I. Unmixed Liquid Layer Over Undisturbed NC Layer}

First the ball weight is measured in the tank head space and the value is recorded. The ball reference position should also be established. Testing for pulley friction and load cell calibration should also be performed at this time. Values for the load cell calibration and the ball weight and pulley friction should be compared to reference values established earlier, so that changes in the performance of the instrumentation can be determined. After system checkout and calibration tests, we are ready for testing in the convective layer. After being lowered into the liquid layer, the effective weight of the ball is again measured to determine the liquid density. The ball will be lowered at slow velocity (10 $\mathrm{cm} / \mathrm{s}$ ) through the entire layer until the sludge interface is reached. It is important to first determine the depth to the NC layer. At intervals of about $1 \mathrm{ft}$. density measurements are obtained throughout 


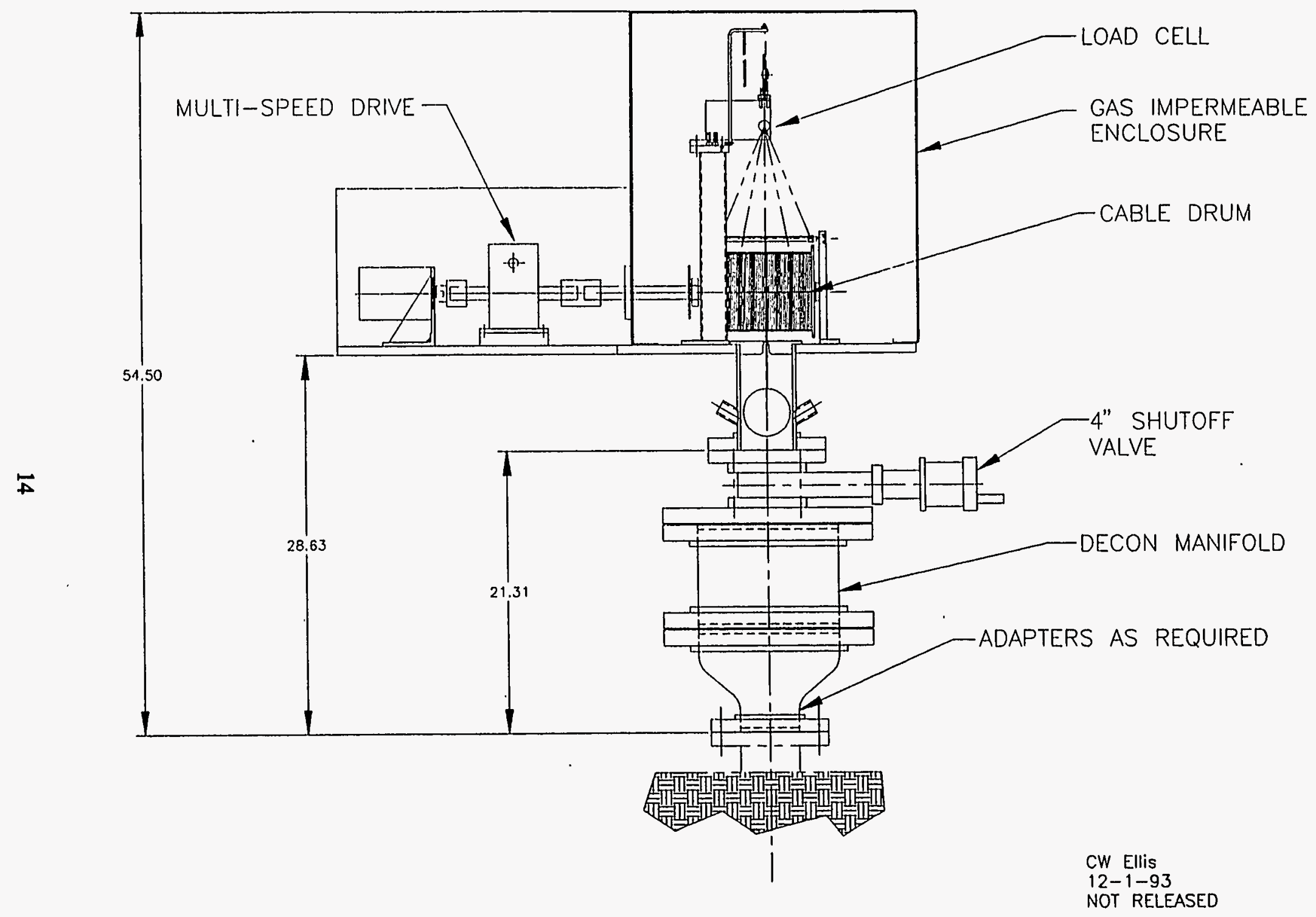

Figure 6. Diagram of In-Tank Apparatus 
the layer. The sludge layer will be evidenced by a reduction in the cable tension to values which may approach zero as the ball is lowered at a constant velocity of $10 \mathrm{~cm} / \mathrm{s}$. When the tension is observed to drop in some dramatic fashion, the ball velocity should be reduced to $1 \mathrm{~cm} / \mathrm{s}$ and the cable tension should be recorded as the ball is dropped at this velocity. If the cable tension approaches zero (adjusted for cable and pulley weight), the ball velocity should be reduced to $0.1 \mathrm{~cm} / \mathrm{s}$. If the cable tension remains at near zero, then the velocity should be dropped to the minimum possible value, about $0.02 \mathrm{~cm} / \mathrm{s}$. If the cable tension does not increase, the ball is being supported by the NC layer and will not sink. If a non-zero reading was obtained at any of the above velocities, then the ball will sink. The above steps are necessary in order to determine a suitable cable tension for the initial drop into the NC layer. We now retrieve the ball until the tension approximates the last value measured under static conditions in the $\mathrm{C}$ layer. The position of the ball where this occurs defines the liquidsludge interface. The position is recorded, and at this time the density profile of the liquid layer is also known. The ball is retrieved about $20 \mathrm{~cm}$ and then the ball is retrieved at high velocity $(1 \mathrm{~m} / \mathrm{s})$ and a measurement of the drag force is obtained to determine the viscosity of the fluid. The same measurement is repeated under falling conditions with the ball descent rate at $1 \mathrm{~m} / \mathrm{s}$. The ball is stopped before it reaches the sludge layer. Both the raising ball and the falling ball tests should then be repeated, so that the ball is returned to near the top of the $C$ layer. Due to the fact that the maximum ball velocity is limited to about $1.5 \mathrm{~m} / \mathrm{s}$, it is not possible or advisable to perform actual free fall tests in the $C$ layer where some actual terminal ball velocity is approached. Measurements with the $\mathrm{C}$ layer are now complete.

Assume for the moment that the ball will sink under its own weight. The ball is then lowered to the NC layer and a force is chosen which will be maintained as the ball is lowered at variable velocity. This force is the first non-zero value measured during our initial tests in the NC layer where restraining force was measured at different velocities. Let the ball sink as far as it can, even to the bottom, and record the velocity as a function of position. The variation in the velocity with a constant restraining force will provide very useful information about the inhomogeneities of the waste.

The technique described immediately above may have some difficulties for waste which has significant inhomogeneities, particularly if the yield strength varies considerably as a function of depth. If the NC layer has even a modest yield stress, most of the drag force on the ball is due to the yielding of the material. To maintain a constant restraining force in changing yield conditions, the ball velocity must undergo large changes. It may not be possible to maintain the constant force condition. If this is observed to be the case, then on repeat runs which are performed later, we should hold the velocity constant at a low value $(0.1 \mathrm{~cm} / \mathrm{s})$ and record the variation in the restraining force.

If the ball will not sink under its own weight, then we must be prepared to physically sink the ball using a long rod which is manually pushed from the surface. No data should be collected during this operation, except the amount of cable released from the spool has to be accounted for.

With the ball near the bottom of the tank, either by sinking under its own weight or by manual assistance, we can then begin experiments using retrieval of the ball. We should attempt to collect data at a minimum of five different velocities which span as large a velocity range as possible. Current designs allow the ball to be controllable from a maximum of $1.3 \mathrm{~m} / \mathrm{s} \mathrm{down}$ to $0.02 \mathrm{~cm} / \mathrm{s}$. Our first velocity should be $0.05 \mathrm{~cm} / \mathrm{s}$. It is presently not clear how long or how far we should pull the ball in order to obtain steady-state flow conditions about the ball. Further laboratory testing with similar diameter balls in heated simulants may clarify this issue. For the moment we will adopt results already found in the laboratory with simulants. Tests performed in the laboratory show that 
we will typically reach steady-state flow conditions after about $10 \mathrm{~cm}$. Therefore tests at each velocity should be performed for time intervals such that $15 \mathrm{~cm}$ of distance is traveled. The second velocity should be $0.1 \mathrm{~cm} / \mathrm{s}$, followed by $0.5 \mathrm{~cm} / \mathrm{s}$ and then $1.0 \mathrm{~cm} / \mathrm{s}$ and finally $2.0 \mathrm{~cm} / \mathrm{s}$. Thus, a single complete test will require a distance of about $75 \mathrm{~cm}$ (or $2.5 \mathrm{ft}$.) for performance. Operator judgement will be required in determination of when a steady-state condition has been reached and in setting the pull distance on any particular set of tests. If additional modeling shows that velocities in excess of $2 \mathrm{~cm} / \mathrm{s}$ can provide useful information, then testing may also be performed at higher velocities.

At the end of each drag test, we will perform measurements to determine the local density of the NC layer. Thus, density measurements occur after each $15 \mathrm{~cm}$ pull at some given velocity. The ball should be oscillated in order to fluidize the waste around the ball and then a static measurement should be performed. Additional laboratory testing is necessary to determine the parameters of the oscillation. However, the general procedure is fairly clear and we have approximate ideas concerning frequency and amplitude. First the ball should be lowered about $3 \mathrm{~cm}$ into the region through which the ball has already passed. Then the ball should be oscillated at an amplitude of about $2 \mathrm{~cm}$ (peakto-peak) at a frequency of about $0.1 \mathrm{~Hz}$. While we can control the pulling of the ball, the settling relies on gravity. The ball should be pulled up $2 \mathrm{~cm}$ in about 4 seconds, then allowed to settle for 6 seconds (same deceleration and same amount of line played out) and then the process is repeated. After five cycles the ball should be retrieved $1 \mathrm{~cm}$ and then a static measurement should be obtained. Force and velocity data should be obtained during the whole process. The prospect of fluidizing the NC layer in this manner has been attempted with some success in the laboratory, but it is not clear that the method will work in the tank. An examination of the data taken during this exercise will shed light on the utility and reliability of the data. Even if fluidization occurs, there is some question as to whether the measurement will provide a true measure of the local waste density. If this method fails, some other technique may have to be developed in order to make this most difficult measurement.

Testing should then be continued throughout the rest of the NC layer proceeding upwards. For the second series of tests, the order of the velocities should be reversed, going from $2 \mathrm{~cm} / \mathrm{s} \mathrm{to} 0.2 \mathrm{~cm} / \mathrm{s}$. Another static measurement should be obtained at the end of the second run. On the third run we should start at the high velocity again and work towards the low velocity, while on the fourth run we should start at low velocity and work towards high velocity. The process should continue until the top of the NC layer is reached, with the order of the velocities following the first four runs. If the $\mathrm{NC}$ layer is $15 \mathrm{ft}$., we should expect about six complete runs. The ball should be immediately returned to the bottom of the tank if it will sink on its own, and then left there until another set of data are required. Repeat experiments should be performed at intervals no closer together than one day in order to examine the reproducibility of results.

Interfaces to and within the NC layer deserve some attention. In traversing the waste, the ball disturbs a volume much greater than the actual ball volume. The effected volume is not yet well known and may depend on rheology as well as ball size. However, as a rule of thumb, the ball will affect material to a distance such that the affected area is about ten times the ball cross-sectional area. Thus material within a radius of about $15 \mathrm{~cm}$ centered on the ball will be influenced by the ball and we can assume that material within $15 \mathrm{~cm}$ above and below ball center will also be influenced. Therefore test results obtained with the ball closer than $15 \mathrm{~cm}$ to the tank bottom, tank wall, or NC-C layer interface should not be considered in data reduction. The influence of layers or interfaces adjacent to one in which the ball is located points out a possible limitation to the usefulness of the ball 
rheometer. As mentioned above, we will require about $75 \mathrm{~cm}$ to perform a test over a suite of velocities. It is implicitly assumed that the waste is homogeneous over the entire $75 \mathrm{~cm}$. If the waste is not uniform then the derived rheological results will suffer in accuracy. The nature of the inhomogeneity is important. If the inhomogeneity is random with small deviations about some average values, then we should still expect good average results with increased uncertainties. If the NC layer is actually made up of many layers where the rheology changes in the same direction from top to bottom, then we will have considerable difficulty in reducing the data unless somehow these changes are accounted for. Thus, it is very important to understand the nature of the waste as soon as possible, and this is why the first test described above where the ball traverses the waste either at constant velocity or under constant force is planned. While the presence of inhomogeneities is lamentable from a data reduction point of view, it should be remembered that on some higher plane what we are really after is an accurate characterization of the waste. If the ball rheometer can show that the waste is inhomogeneous, then we have collected valuable information about it, regardless of our ability to reduce the data to accurate rheological parameters.

\subsubsection{Case II. Mixed Layer Above Unmixed Sludge Layer}

This case represents a very important use for the ball rheometer, perhaps the most important. The data obtained under these conditions not only help determine waste rheological properties but also will be used to determine the effectiveness of the mixer pump. Looking longer term, it is possible that the ball rheometer may also become a primary diagnostic for judging the effectiveness of the sonic probe or other mitigation techniques.

If the $\mathrm{C}$ layer becomes fairly thoroughly mixed with the NC layer, it can be expected that it will have a very low yield strength in addition to a low viscosity. We will attempt measurement of these properties, but they may be difficult owing to an expected low drag force, especially at low ball velocities. Probably the most important measurement which we can perform in the mixed region is that of effective fluid density, which we should be able to measure to good precision. From this single measurement we can obtain an idea of the solids distribution within the mixture and, if measurements are performed over time, the solids settling rate.

The procedure begins as in Case I above. We measure the weight of the assembly in the tank dome space first and then immerse the ball within the liquid and perform density measurements throughout the mixed layer at about $1 \mathrm{ft}$. intervals. If there is a nonconvective layer, we will find it as before and we will then position the ball at the top of the NC layer and begin tests in the C layer with the ball in retrieval mode. At this point the procedure may change from that followed in Case $\mathrm{I}$. The rheological properties may not be consistent throughout the entire layer. First we should pull the ball at $1 \mathrm{~m} / \mathrm{s}$ until we reach the top of the layer. Then we should drop it at $0.6 \mathrm{~m} / \mathrm{s}$ until we reach the $\mathrm{NC}$ layer. Then we should retrieve it at $0.3 \mathrm{~m} / \mathrm{s}$ until we reach the top again. We should then return the ball to the top of the NC layer at $0.5 \mathrm{~m} / \mathrm{s}$ to begin tests in this region. Data should be collected in all of these rising and falling ball tests.

Testing in the NC layer will be conducted as described in Case I. This layer may or may not be very thick, and so it may not be possible to run an extended series of tests where measurements over the same velocity range can be repeated several times. If the ball will sink on its own weight, we will follow the steps described above in Case I. If the ball will not sink (more likely in this case), we should again manually push it to the bottom. However, when measurements are concluded in the NC 
layer, the ball should not be returned to the tank bottom but left in the mixed region. The difficulty associated with sinking the ball combined with the fact that measurements are most useful in the mixed region suggest that this approach is the most favorable.

If testing shows that the NC layer is not very thick, say less than $2 \mathrm{ft}$., there is no point in performing measurements in the NC layer because it will be too difficult to obtain reliable data (due to interface effects). In addition, this volume becomes relatively unimportant in the tank dynamics.

\subsection{System Description}

\subsubsection{Ball}

The ball to be used in this system should be of diameter 3.6 inches $(9.1 \mathrm{~cm})$ and be made from tungsten or a tungsten alloy. The ball diameter is chosen so that it will fit down a nominal 4-in. diameter riser pipe and the material is chosen so that the ball density be as high as practical. Both ball diameter and density are driven to be as high as practical in order that the ball has the greatest likelihood of sinking, under its own weight, into the nonconvective layer, which may possess a yield strength of order $1 \mathrm{psi}$. The ball should be coated with a polyethylene coating of thickness around 0.003 inches in order to reduce crystalline accumulation on the ball. A cable (described below) should be permanently attached to the ball in such a manner that minimal deviation from sphericity is obtained. Other (smaller) ball diameters and densities may be identified as useful from experimentation or modeling results.

\subsubsection{Cable}

The cable must be strong enough to support a weight of $500 \mathrm{lbs}$. Multi-strand stainless steel cable with a diameter of $1 / 8$ in. is appropriate. The diameter of the cable is not critical, and thicker cables are acceptable. However, efforts should be made to keep the cable diameter and weight as small as possible while satisfying the loading requirement, which is critical. Both the extended cable weight and the drag force on the cable must be accounted for in determination of the drag force on the ball. Minimization of these forces is the best approach, and this is accomplished by minimizing the cable diameter and weight. The cable should be supplied with a polyethylene coating in order to prevent crystal build up and facilitate decontamination procedures.

\subsubsection{Load Cells}

The load cells measure about twice the tension in the cable plus the weight of mechanical components, such as the main pulley. The tension is due to the ball weight, extended cable weight, the drag force due to the ball and cable, buoyancy forces, and friction in the main pulley. Due to the design of the instrument, some of these loads are measured at twice their true value. The pulley friction and the weight of the mechanical components are measured at their true values while the weight of the ball and cable, the drag force, and the buoyancy force are measured at twice the true value. Some confusion will result if we are not careful in our discussions to distinguish between actual load and measured weight or load. Unless specifically stated otherwise, we will consider measured loads in the text below. The load carried by the cable will in most cases be much larger than the pulley and supporting structure weight, and we will not concern ourselves with loads other than those carried by 
the cable in the discussion below. In any calculation of ball drag force the weight due to mechanical components and to the main pulley friction must be accounted for.

The load cells should have a total error (from all sources) of less than $0.1 \%$ of full-scale reading. The desired accuracy of the drag force measurements is $1 \%$. Therefore, if the measured drag force (which is twice the actual drag force) is less than $10 \%$ of the rating for the load cell, the load cell should be replaced with one of greater sensitivity (i.e., smaller load rating). The ball will weigh about $16 \mathrm{lb}$ (and therefore have a measured weight of $32 \mathrm{lb}$ ). If the measured drag force is less than $3.2 \mathrm{lb}$, it becomes impossible to measure total force (approximately ball weight plus drag force) with any load cell of the stated accuracy and still maintain the requirement for $1 \%$ error in the drag force. For this reason, it may be necessary, in the case of small drag forces, to counterbalance the weight of the ball. Counterbalancing will be discussed below. If the measured drag force exceeds $5 \mathrm{lb}$, it is not necessary to counterbalance the ball weight, assuming a $50 \mathrm{lb}$ load cell is available.

\subsubsection{Counterbalance Weight}

The use of a counterbalance weight has some attractive features if the drag force which we are trying to measure is quite small (less than several pounds). On the other hand, there are also many instances where a counterbalance weight can be quite annoying and provide little benefit to the measurement procedure. For simplicity of operation and for the minimization of possible mistakes in the data collection process, it is recommended that experiments be performed without the use of a counterbalance if at all possible. The ability to provide counterbalancing should be built into the system, and a simple spring mechanism is suggested. The use of the counterbalance in experiments should be at the discretion of the lead engineer and the counterbalance should only be employed in limited cases where data are unobtainable otherwise.

\subsubsection{Ball Speeds}

For measurements in the nonconvective region, ball velocities in the range from 0.05 to $2.0 \mathrm{~cm} / \mathrm{s}$ are presently required. These ball speeds are required in order to ensure that measurements are made at low Reynolds numbers $(\operatorname{Re}<0.1)$ where theories relating drag force and velocity to rheological properties are available. Continued investigation into model development may show that higher ball velocities can provide additional information, in which case this range of velocities may be extended. The acceptable error is $1 \%$ of the ball velocity. In the nonconvective region the ball velocity will be in the range from 1.0 to $100 \mathrm{~cm} / \mathrm{s}$ with the same error. Higher velocities are required in order to obtain measurable drag forces. Acceleration of the ball to final velocity should be variable (programmable), since it is presently unclear if information is obtainable from drag force data acquired during acceleration. A typical acceleration for measurements in the nonconvective region is $1 \mathrm{~cm} / \mathrm{s}^{2}$ while for the convective or mixed region a value of $10 \mathrm{~cm} / \mathrm{s}^{2}$ would be more appropriate.

For density measurements, the ball will have to be held stationary while measurements of the apparent ball weight are obtained. The apparent weight of the ball will be about $91 \%$ of the true weight of the ball if the fluid density is about $1.6 \mathrm{~g} / \mathrm{cm}^{3}$ and the ball is tungsten. Under the best of circumstances we can expect to be able to measure fluid density to within $2 \%$ in the convective layer. Measurements in the nonconvective layer cannot be expected to be as accurate. Experiments with simulants have to date shown that errors in density measurements are around $10 \%$. This error will increase as the yield stress increases. 


\subsection{Model Development}

The system is equivalent to a falling-ball viscometer commonly used for Newtonian fluids. Similar viscometers also are used for suspensions (see, for instance, Abbott et al. 1991a, and Powell and Walla 1991). A schematic description of the system is shown in Figure 7.

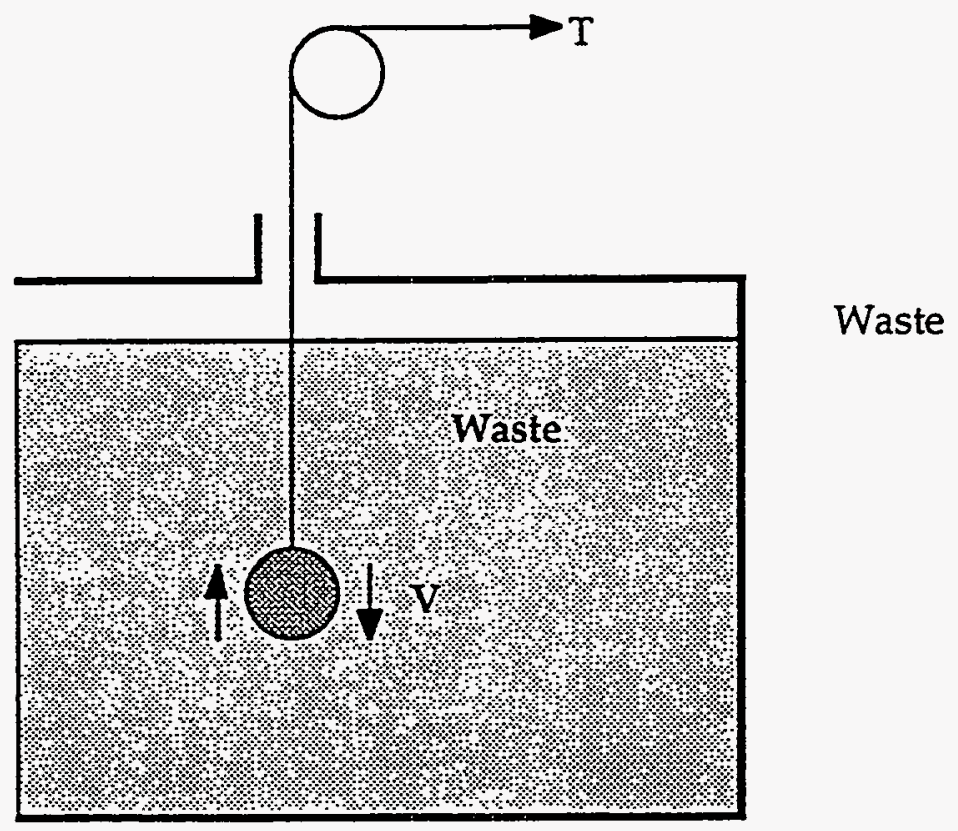

Figure 7. Schematic of the System

The equation of motion for the sphere may be written as

$$
M a=W-B-D-T-L,
$$

where $M$ is the mass, $a$ is the acceleration, $W$ is the weight, $B$ is the buoyancy force, $D$ is the drag force, $T$ is the external force shown in Figure 7 , and $L$ is the system losses (depending on the number and arrangement of the pulleys).

If the sphere is moving with a constant velocity (V), Eq. (1) reduces to

$$
T=W-B-D-L
$$

Finally, if the sphere is stationary, the drag force in Eq. (2) vanishes, and the equation reduces to 


$$
T=W-B
$$

In this system, the weight (W) of a given sphere is known. Also, it is assumed that the frictional losses of the system (L) can be obtained from a calibration test for the final system design. The measurable parameters are the external force $\mathrm{T}$ and the motion parameters of the sphere (a and/or $\mathrm{V}$ ) (using the pulley's rotational speed, for example). The required data are the waste density $(\rho)$ and the waste viscosity $(\mu)$. If applicable, the waste yield strength $(\tau)$ is another parameter that can be reduced from the above data. Obviously, none of the desired parameters are measured directly during the test. Therefore, they must be obtained by using models from the measurable parameters.

\subsection{Data Reduction Methodology}

In order to transform measured quantities (force and velocity) into fluid properties, we need to use physical models which form the relationships. The relationships depend upon the type of fluids which we are dealing with. Fluid types which we will consider include Newtonian fluids and several nonNewtonian fluids including Bingham plastic, generalized Bingham plastic, and power law fluids. It is presently believed that the convective layer can be adequately represented as a Newtonian fluid while the nonconvective layer can be approximately characterized as a generalized Bingham plastic. However, these beliefs may change as more information is learned about the waste. In any case, the drag force on the ball at some particular velocity depends on the nature of the fluid. In this section, we will outline how the drag force is related to fluid properties for the fluid types mentioned above. It should be noted that the theories about to be presented are all steady-state theories and do not consider transient behavior. If after further review it is learned that none of the models which will be described below are applicable to this problem, then another model must be found. Other models are available, and those discussed here should not be thought of as comprising all possibilities. Presently it is believed that one of them should be suited for our case.

The measured data must be converted to density, viscosity, and yield strength through models. The density can be obtained from Eq. (3), knowing the weight $W$ and having measured the force $T$. The waste density $\left(\rho_{w}\right)$ can be obtained as

$$
\rho_{w}=\frac{6(W-T)}{\pi d^{3} g},
$$

where $d$ is the sphere diameter. However, we must note that this equation is not valid if some of the weight is carried by the material's yield strength in the NC layer. To measure the true buoyancy, we must fluidize the material around the sphere. The motion of the sphere may be sufficient to fluidize the material. However, if the material regains its strength very quickly after the motion ceases, this method will not work. Furthermore, if the motion results in accumulation of a liquid layer around the sphere as the solids migrate away or if the solids are not freely suspended in this liquid, the measured density will not represent the actual density of the slurry.

Currently, the sphere must be designed to fit through one of the smaller risers (4-in. nominal ID) in Tank 101-SY. Thus, the diameter of the sphere would be approximately 3.6 in. The corresponding 
weight of a tungsten sphere of this size is $76 \mathrm{~N}(17 \mathrm{lbf})$. The waste density is expected to vary between 1400 and $1600 \mathrm{~kg} / \mathrm{m}^{3}$. Thus, the net weight inside the waste would be between 70.5 and $71.3 \mathrm{~N}(15.9$ and $16 \mathrm{lbf})$. Therefore, to measure the waste density accurately within this range, the load cell must be sensitive to variations less than $0.1 \mathrm{lbf}(0.6 \%$ of the total weight). Thus, the load cell must be sensitive to variations at least an order of magnitude smaller than this value if we intend to do an accurate density measurement in the range of 1400 to $1600 \mathrm{~kg} / \mathrm{m}^{3}$.

The capability of quantifying the drag force $\mathrm{D}$ in the above equations is key to obtaining the rheology parameters, such as viscosity and yield strength. The drag force can be written in terms of a drag coefficient $(\mathrm{Cd})$ as

$$
D=\frac{1}{2} \rho_{w} C_{d} V^{2}\left(\frac{\pi}{4} d^{2}\right)
$$

However, converting the measured drag force and the velocity to viscosity requires knowledge of the type of fluid.

\subsubsection{Newtonian Fluids}

For a Newtonian fluid, the drag coefficient of a sphere is well quantified (see, for instance, Gerhart and Gross 1985) as a function of the Reynolds number (Re), defined as

$$
R e=\frac{\rho_{w} V d}{\mu} .
$$

The quasi-steady drag coefficient of a sphere is given by

$$
C_{d} \approx \frac{24}{R e}+\frac{6}{1+\sqrt{R e}}+0.4
$$

for $\operatorname{Re}<2 \times 10^{5}$.

Figure 8 shows the drag force on a sphere moving in a Newtonian fluid. As indicated by this figure, the expected drag forces when the sphere is in the $C$ layer are very small for the velocity and viscosity range of interest. Likewise, if we set a constant drag force, the resulting terminal velocities would be very large unless the drag force was set to a very small value. Note that if we operate at a high $R e$ range, $C_{d}$ approaches a constant, independent of $\mu$. Thus, the drag data in this range cannot be used to obtain viscosity information. For best accuracy, we want to operate in the Stokes regime ( $\operatorname{Re}<0.1$ ), where $C_{d}=24 / \operatorname{Re}$. For a fluid with a $100-\mathrm{cP}$ viscosity, $\operatorname{Re}=0.1$ corresponds to a velocity of less than $0.07 \mathrm{~mm} / \mathrm{s}\left(\mathrm{d}=0.1 \mathrm{~m}\right.$, and $\left.\rho=1500 \mathrm{~kg} / \mathrm{m}^{3}\right)$. The corresponding drag force would be $\approx 10^{-6} \mathrm{~N}\left(3 \times 10^{-7} \mathrm{lbf}\right)$. For such fluids, the required accuracy of the velocity and drag measurements are likely to be extremely high. 


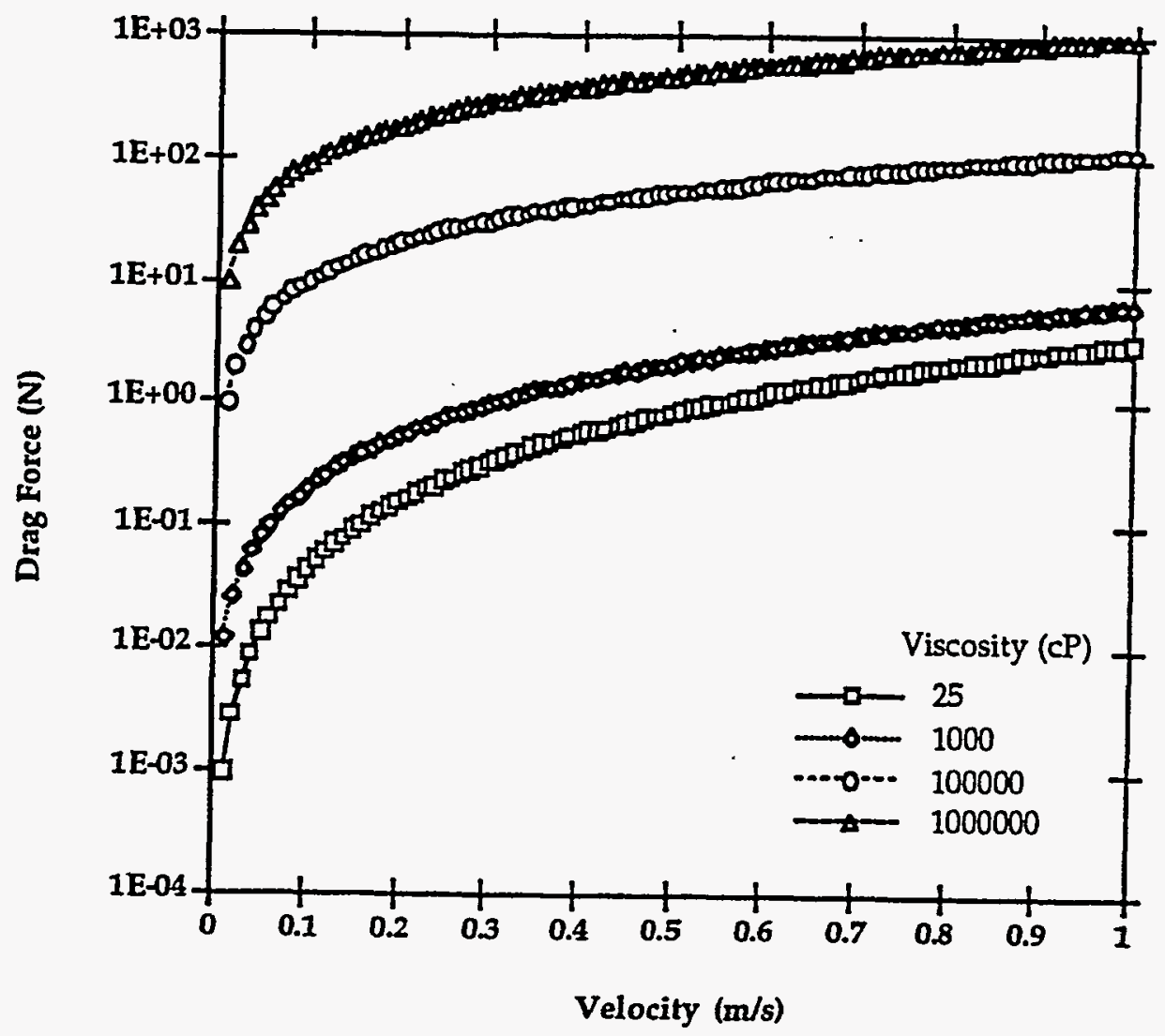

Figure 8. Drag Force on a Sphere with a Diameter of $0.1016 \mathrm{~m}$ in a Newtonian Fluid for Various Viscosities

While the above methodology is only applicable for Newtonian fluids, we can use it to obtain an approximate estimate for the drag forces anticipated in the NC layer, which is believed to be nonNewtonian. If we use a constant apparent viscosity of $10^{5}$ and $10^{6} \mathrm{cP}$, the resulting drag forces also are shown in Figure 8. As shown, the drag forces can be quite large for such high viscosities. Note that for a 3.6-in. diameter tungsten sphere, the net weight inside the waste is roughly $70 \mathrm{~N}$ (16 lbf). Thus, the terminal velocity of a falling ball corresponding to this value of the drag force would be less than $10 \mathrm{~cm} / \mathrm{s}$ using a $10^{6}-\mathrm{cP}$ viscosity material.

\subsubsection{Bingham Plastics and Generalized Bingham Plastics}

The most common viscoplastic fluid is known as the Bingham plastic. The simplest fluid model for the Bingham plastic model can be described by the following equation: 


$$
\begin{aligned}
& \tau=\tau_{B}+\mu_{B} \gamma \quad \text { for } \tau>\tau_{B} \\
& \gamma=0 \text { for } \tau \leq \tau_{B}
\end{aligned}
$$

where $\tau_{\mathrm{B}}$ is the Bingham yield stress, $\mu_{\mathrm{B}}$ is the plastic viscosity, and $\gamma$ is the shear rate.

If a viscoplastic material shows a shear thinning, then the fluid behavior can be described by the Herschel-Bulkley model. Based on knowledge obtained about the waste to date, this model seems to be the most appropriate for a description of the NC waste. The Herschel-Bulkley model is a generalization of the simple Bingham model and replaces the linear shear rate dependence with a power law behavior. Thus, three parameters are needed to describe the fluid:

$$
\begin{aligned}
& \tau=\tau_{B}+\mu_{o} \gamma^{n} \quad \text { for } \tau>\tau_{B} \\
& \gamma=0 \text { for } \tau \leq \tau_{B}
\end{aligned}
$$

where $\mu_{\mathrm{o}}$ is the consistency index (or zero-shear rate viscosity), and $\mathrm{n}$ is the power law index.

The drag force on spheres for Bingham plastics has been investigated experimentally. Analytical studies based on the solution of field equations also exist. A good summary of these works was recently reported by Chhabra (1993).

There are several empirical correlations formulating the functional relationship between the drag coefficient and pertinent dimensionless numbers. The choice of dimensionless parameters depends on the fluid model. For Bingham plastics, the important dimensionless number is the Reynolds number $\left(\operatorname{Re}_{\mathrm{B}}\right)$, based on the Bingham viscosity $\left(\mu_{\mathrm{B}}\right)$ and the Bingham number $\left(\mathrm{N}_{\mathrm{B}}\right)$. In the case of generalized Bingham plastics (Herschel-Bulkley fluid model), these two numbers are modified to include the consistency $\left(\mu_{\mathrm{o}}\right)$ and power law $(\mathrm{n})$ indices to consider the power law nature of the shear-stress shearrate relationship. Collapsing the family of drag coefficient curves into a single curve using a twodimensionless number (Bingham and plastic Reynolds numbers) has been attempted. This has been accomplished successfully in some cases.

An earlier correlation was provided by Andres (see Chhabra 1993) and was given as

$$
C_{d} \operatorname{Re}_{B}^{1.8}=10^{12.26-0.78 \mathrm{He},}
$$

where $\mathrm{Re}_{\mathrm{B}}$ and $\mathrm{He}$ are the Bingham Reynolds number and the Hedstrom number is defined as 


$$
\begin{aligned}
R e_{B} & =\frac{\rho V d}{\mu_{B}} \\
H e & =\frac{\tau_{B} d^{2} \rho}{\mu_{B}^{2}} .
\end{aligned}
$$

The applicability of this correlation is limited with the range $1 \leq \mathrm{He} \leq 27$.

Pazwash and Robertson (see Chhabra 1993) express the difference between the values of drag coefficient for a Bingham fluid and that of a Newtonian fluid $\left(C_{d N}\right)$ as

$$
C_{d}-C_{d N}=36 \frac{H e}{R e_{B}^{2}}
$$

This correlation is given for a range of $920<\mathrm{He}<3600$ and $60<\mathrm{Re}_{\mathrm{B}}<2000$. Equation (11) gave results in reasonable agreement with Ansley and Smith's and Pazwash and Robertson's data but overestimated the data of Valentik and Whitmore (Chhabra 1993).

Plesis and Ansley (Chhabra 1993) proposed a dynamic parameter of

$$
Q=\frac{R e_{B}}{1+N_{B}}
$$

for a range of $0.7<\mathrm{Re}_{B}<1200$ and $2.9<\mathrm{N}_{B}<16.5$. The Bingham number $\mathrm{N}_{B}$ is defined as

$$
N_{B}=\frac{\tau_{B} d}{\mu_{B} V}
$$

The drag coefficient was expressed as

$$
C_{d}=5 Q^{-0.49}
$$

As can be seen, this relationship does not approach Newtonian behavior as $N_{B}$ goes to zero.

Later, Ansley and Smith (1967) modified this relationship and gave the dynamic parameter as 


$$
Q=\frac{R e_{B}}{1+\frac{7 \pi}{24} N_{B}} .
$$

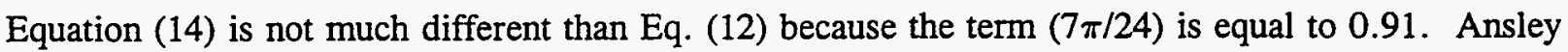
and Smith gave the drag coefficient as

$$
C_{d}=\left\{\begin{array}{cc}
\frac{24}{Q} & \text { for } Q<20 \\
0.4 & \text { for } Q>20
\end{array}\right.
$$

Equation (15) gave reasonable results for the data of Atapattu (1989) and Valentik and Whitmore but underpredicted the data of Pazwash and Robertson (Chhabra 1993).

The dynamic parameter, $Q$, is redefined by Hanks and Sen for a generalized Bingham plastic (see Chhabra 1993 for the suggested equation). Atapattu (1989) (see Chhabra 1993) suggested that Eq. (11) be used if the $\operatorname{Re}_{B}$ and $N_{B}$ are redefined to consider the power law contribution for generalized Bingham plastics. He gave the drag coefficient as

$$
C_{d}=\frac{24}{Q} \text {. }
$$

where $Q$ is defined as

$$
Q=\frac{R e_{P L}}{1+K N_{B, H B}} .
$$

The plastic Reynolds number $\left(\mathrm{Re}_{\mathrm{PL}}\right)$ and Herschel-Bulkley Bingham number $\left(\mathrm{N}_{\mathrm{B}, \mathrm{HB}}\right)$ are defined as

$$
R e_{P L}=\frac{d^{n} V^{2-n} \rho}{\mu_{o}} \text { and } N_{B, H B}=\frac{\tau_{B} d^{n}}{\mu_{o} V^{n}} \text {. }
$$

Equation (16) predicted Atapattu's own data, with average and maximum deviations of $30 \%$ and $48 \%$.

Ito and Kajiuchi (1969) presented the drag force as a function of generalized Reynolds and Bingham numbers. There are additional studies reporting drag coefficients in Bingham plastics (Brookes and Whitmore 1968, 1969).

Direct solution of the field equation received very little attention. One of the earlier studies was reported by Yoshioka and Adachi (1971) and Adachi and Yoshioka (1973). Both papers used the stress and velocity variational principles to obtain upper and lower bounds on the drag coefficient. The calculated shape of the sheared zone was somewhat different than that suggested by Ansley and 
Smith (1967) and calculated by Beris et al. (1985). The estimated drag coefficient was tabulated as a function of the Bingham number. Chhabra (1993) compared his results with Ansley and Smith's data. This comparison indicated that Yoshioka and Adachi's (1971) upper-bound prediction agreed with Ansley and Smith's data.

Bhavaraju et al. (1978) obtained the drag coefficient for a bubble in Bingham plastics, as well as in power law fluids. Their analysis was based on a perturbation analysis in which the stream function, velocity, and deformation tensor were expressed in the form of a power series for the perturbation parameter of the Bingham number. A first-order solution gave the following equation for the drag coefficient:

$$
C_{d}=\frac{16}{R e_{P L}}\left(1+3.22 \frac{N_{B}}{2}\right) .
$$

The term in parentheses can be assumed as the correction factor for the drag coefficient.

A more complete solution of the field equations was reported recently by Beris et al. (1985). They present a finite-element/Newton method for a sphere falling in a Bingham fluid under a gravitational field. They used the calculated variational integrals suggested by Yoshioka and Adachi (1971) to verify the numerical results.

Beris et al.'s (1985) results indicated significant differences with the earlier approximate calculations. The predicted shape of the sheared region was similar to the description by Ansley and Smith (1967). The small unsheared region at $0^{\circ}$ and $180^{\circ}$ angles increased by increasing the yield stress parameter Yg, defined as

$$
Y_{g}=\frac{\pi d^{2} \tau_{B}}{2 F}
$$

which reduces to

$$
Y_{g}=\frac{3 \tau_{B}}{d \Delta \rho g},
$$

for a sphere sinking under the gravity force. In Eq. (19b), $\Delta \rho$ is the density difference between the sphere and the fluid.

In Eq. (19a), $\mathrm{F}$ is the external force acting on the sphere. The typical tangential velocity profile at a $90^{\circ}$ angle was steeper for higher yield stress parameters and tended to follow the Newtonian profile as $Y_{g}$ went to zero. 
They presented the calculated drag coefficient as a function of the Bingham number and a yield-stress parameter. Their results indicated that the drag coefficient tended to infinity (sphere stops moving) as $Y_{g}$ goes to 0.143 . The numerically computed $Y_{g}$ reached nearly a constant slope in the $C_{d}-Y_{g}$ plane, except for very small values of $Y_{g}$. The calculated drag coefficient agreed reasonably with Ansley and Smith's data and the upper bound of Adachi and Yoshioka's (1973) prediction.

By fitting the numerical results, the drag coefficient is expressed as

$$
C_{d} \approx 1+1.87 \sqrt{N_{B}}+1.152 N_{B}
$$

More recently, Atapattu (1989) (see Chhabra 1993) used an optical technique and carried out quantitative and qualitative flow visualization experiments. He formulated the additional effect in the presence of a wall. The calculated shape of the sheared zone resembled qualitatively the previously postulated ones by Ansley and Smith (1967) and Beris et al. (1985). Atapattu's drag prediction was given by Eqs. (16) and (17).

The Reynolds number can be written as

$$
R e_{B}=\frac{\rho V^{2}}{\mu_{B} V / d}
$$

where the numerator is the dynamic pressure and the denominator is the shear stress. A more general form of the Reynolds number, which includes the yield stress, is therefore

$$
\operatorname{Re}_{B}=\frac{\rho V^{2}}{\tau}
$$

This definition may be more meaningful since the classical Reynolds number considers only the plastic viscosity. Often in this case the Hedstrom number is introduced as another correlation parameter to account for the yield stress. Dedegil (1989) compared this theory with Valentik and Whitmore's data and obtained a single drag coefficient curve as a function of Reynolds number. He suggested the following equations for spheres moving through a Bingham fluid. 


$$
\begin{aligned}
& C_{d}=\frac{24}{R e_{B}} \quad R e_{B}<8 \\
& C_{d}=\frac{22}{R e_{B}}+0.25 \quad 8<R e_{B}<150 \\
& C_{d}=0.4 \quad R e_{B}>150
\end{aligned}
$$

where

$$
R e_{B}=\frac{\rho V^{2}}{\tau+\mu_{B} V / d}
$$

Tran et al. (1993) conducted experiments and used Dedegril's suggestions to correlate the drag coefficient for Bingham fluids containing particles. Their results indicated that this formulation correlates the data very well.

\subsubsection{Power Law Fluids}

The drag force on a sphere moving in power law fluids has been investigated by various authors. The viscosity of a power law fluid is defined as

$$
\mu=\mu_{0} \gamma^{n-1},
$$

For a detailed discussion of this subject, readers are referred to the studies of Chhabra (1983), Dazhi and Tanner (1985), and the textbook by Chhabra (1993). Theoretically, converting a drag force and velocity measurement into a viscosity model would require a finite element code [similar to that discussed by Dazhi and Tanner (1985)]. However, such numerical solutions already exist for the entire range of power law indices (n), ranging from 0 to 1 (see, for instance, Dazhi and Tanner 1985). A curve fit to such solutions can be used if we determine that the fluid of interest behaves as a power law fluid.

Because a power law fluid requires two parameters to be quantified ( $\mu_{0}$ and $n$ ), a minimum of two pair of data are required for velocity and drag force. It is also important to note that there appears to be a $\pm 30 \%$ gap between the numerical predictions of the drag force and the experimental data. Not all of the blame for this discrepancy is on the theoretical model. There are a number of uncertainties associated with the experimental data reported in the literature. Primarily, wall effects, viscoelastic effects, and the power law approximation result in experimental uncertainties. Nevertheless, there are some empirical data, and an empirical model may also be used to convert the measured velocity and drag force into a viscosity model. Using either a numerical model or an empirical model, the drag coefficient for a power law fluid can be obtained in the following form: 


$$
C_{d}=F\left(\operatorname{Re}^{\prime}, n\right)
$$

where $\mathrm{Re}^{\prime}$ is the generalized Reynolds number (accounting for the power law index).

Available studies to evaluate Eq. (26) can be classified into two categories: theoretical studies and empirical correlations. Theoretical studies consider the momentum equation for a creeping flow. They employ one of three methods: the variational principles method, the linearization (or perturbation) method, and the extended moment method. Details of these methods are not presented in this report. For more information, the reader should refer to Chhabra (1993).

There is a significant amount of experimental data in the literature. These data are listed by Chhabra (1993). The rheological behavior of test fluids was approximated by the Ellis model, as well as the Carreau model. From the comparison of experimental data and model predictions, using the Carreau fluid model gave very good results. By introducing the notion of a reference viscosity coupled with simple dimensional consideration, Bush and PhanTien (see Chhabra 1993) gave the following approximated theoretical expression:

$$
Y=\left(1+k^{2} A^{2}\right)^{(n-1) R},
$$

for a range of $1>n>0.4$ and $0<\mathrm{A}<400$. In Eq. (27), $\mathrm{Y}$ is the drag correction factor to be applied to the Stokes formula, $\mathrm{A}$ is the Carreau number defined as

$$
A=\frac{2 \lambda v}{d}
$$

$\lambda$ is the Carreau model parameter, and $\mathrm{n}$ is the power index.

Chhabra et al. (1981) indicated that the following expression correlated the values of the drag correction factor within $15 \%$ scatter:

$$
Y=\left[1+0.5 E l^{1.65}(1-\alpha)^{0.38}\right]^{-0.35}
$$

for a range of $1<\mathrm{a}<3.22$ and $0.1<\mathrm{El}<141$. In Eq. (28), El is the Ellis number defined as

$$
E l=\frac{1.4 \mu_{o} V}{d \tau_{1 / 2}},
$$


$\alpha$ is the Ellis model parameter that is a measure of the extent of shear thinning behavior, $\tau_{1 / 2}$ is the model parameter that denotes the value of shear stress at which the apparent viscosity has dropped to $\mu_{0} / 2$ ), and $\mu_{0}$ is the zero shear viscosity.

One important feature of the available studies is that they are all applicable to Stoke's regime, where the flow velocity is small. Consequently, this restriction should be kept in mind when using the waste viscometer data. At this time it should also be understood that there are a whole host of possible numbers and parameters with which a fluid can be characterized, depending on the applicable model. The flow Reynolds number is probably the most important parameter. In the NC layer the Reynolds number will be small, less than or about 0.1 , so that we will operate in the Stoke's regime. In the mixed or C layer the Reynolds number will be from 100 to 1000, well outside the Stoke's regime.

Similar additional studies exist in the literature. The reader is referred to the study of Zhu and Satish (1992) on this subject as an additional reference.

\subsection{Error Analysis}

We essentially need to measure two quantities, the ball velocity and the drag force on the ball. For static measurements, we need the apparent weight of the ball. Velocity is measured by measuring the rotational velocity of the cable spool by use of an encoder. In order to obtain the ball velocity the spool diameter and the cable diameter must be known. All quantities can be measured to high precision and there is little doubt that we can measure velocity to within $1 \%$ over the range from 0.02 $\mathrm{cm} / \mathrm{s}$ to $1 \mathrm{~m} / \mathrm{s}$. Position will be determined from the velocity measurement and should also be known to sufficient precision ( 1 ball diameter). Cable stretch due to loading has not been accounted for in position determination and remains as an open question. However, the error is expected to be relatively unimportant. Thus, we expect no difficulty in measurements of velocity or in the subsequent determination of ball position within the tank.

Errors in determination of the drag force on the ball and the apparent weight of the ball while immersed in the fluid are more complicated. The drag force on the ball will be some fraction of the total tension measured in the cable. We must account for other factors which affect the total load measured with the load cell. These include:

1. Pulley friction - may be a function of load and speed

2. Weight of the spooled out cable - depends on ball position

3. Buoyancy of waste -- depends on density and rheology - acts on ball and cable

4. Drag force on cable

5. Weight of pulley assembly supported by load cell

Some of these factors can be accounted for quite accurately and very simply in laboratory measurements, such as pulley friction, cable weight, and pulley weight. The drag force on the cable and the buoyancy force on the ball cannot be determined from in-tank measurements. Drag force on the cable can be estimated from tests with simulants and some results have already been obtained. In tests with a chemical simulant we have found that the drag force on a cable of about $1 / 8$-in. diameter is about $1 \mathrm{~N} / \mathrm{m}$. The drag force in the same simulant on a 1-in. ball is about $2 \mathrm{~N}$, so that for a 3.6- 
in. ball we expect a ball drag force of about $30 \mathrm{~N}$. We can then expect that even at the deepest point in the tank (about 5 meters into the NC layer) the drag force due to the cable will be less than $20 \%$ of the drag force on the ball. Since the cable precedes the ball in raising ball tests, a part of the fluid will have already been sheared by the cable when the ball passes through it. The magnitude of this effect on the measurements has not been investigated and remains an issue which will be addressed in further laboratory investigations. It is expected that the effect will be small, but an accurate estimate of it is necessary in the reduction of the data.

The buoyancy force on the ball or cable when the ball is being pulled through the NC layer is difficult to determine from any experiment. Perhaps the best we can do is assume that this force can be calculated from a knowledge of the fluid density and the total cable and ball volume involved. It cannot exceed this value. Fully deployed, the cable volume in the waste will be about $15 \%$ of the total (cable plus ball) volume occupied. The buoyancy force for the volume involved in a fluid of specific gravity 1.6 is about $7 \mathrm{~N}$. The error in determining the buoyancy force is the most serious.

It should be noted that any errors made in the above quantities will occur systematically in all measurements. In other words, if the determined drag force measured at one ball velocity is in error due to inadequate compensation of the above quantities, then the drag force measured at a second or subsequent velocity will contain the same error. Such errors will result in an overall error in the determined yield strength of the waste, but will not affect the determined viscosity appreciably. At worse the total systematic error will be less than $10 \mathrm{~N}$, so that if the drag force on the ball is $30 \mathrm{~N}$ or greater, the error in the determined yield stress would be less than $30 \%$, which is acceptable.

It is presently not known exactly how errors in measurement of drag force and velocity will affect the final determination of rheological properties. The model relating these measurements to yield stress and viscosity as a function of strain rate has yet to be developed. If the waste fluid behaves nearly as an ideal Bingham plastic, then we can use the formulation developed by Beris et al. (1985) to obtain some idea of the effects of measurement errors. The expression relating the near-zero shear rate plastic viscosity and the yield stress to the drag force and ball velocity is:

$$
T=6 \pi \mu_{o} R V\left[1+2.65 \sqrt{\frac{\tau_{o} R}{\mu_{o} V}}+2.30 \frac{\tau_{o} R}{\mu_{o} V}\right]
$$

where

$\mathrm{T}$ is the drag force

$\mu_{0}$ is the plastic viscosity

$\tau_{\mathrm{o}}$ is the yield stress

$\mathrm{R}$ is the ball radius

$\mathrm{V}$ is the ball velocity.

In order to obtain both yield stress and viscosity, measurements at two velocities are required, in which case, 


$$
\begin{aligned}
& T_{1}=6 \pi \mu_{o} R V_{1}\left[1+2.65 \sqrt{\frac{\tau_{o} R}{\mu_{o} V_{1}}}+2.30 \frac{\tau_{o} R}{\mu_{o} V_{1}}\right] \\
& T_{2}=6 \pi \mu_{o} R V_{2}\left[1+2.65 \sqrt{\frac{\tau_{o} R}{\mu_{o} V_{2}}}+2.30 \frac{\tau_{0} R}{\mu_{o} V_{2}}\right]
\end{aligned}
$$

where $T_{1}$ is obtained with velocity $V_{1}$ and $T_{2}$ is obtained with velocity $V_{2}$.

For a generalized Bingham plastic, at least three measurements will be required. We have performed calculations to determine the expected drag force on a ball for a range of ball velocities, viscosities, and yield stresses. The results are shown in Table 1 . The units for all quantities are cgs, so viscosity is in poise, yield stress is in dynes $/ \mathrm{cm}^{2}$, velocity is in $\mathrm{cm} / \mathrm{s}$, ball radius is in $\mathrm{cm}$, and drag force is in dynes. In Table $1, T_{1}$ goes with $V_{1}$ and $T_{2}$ goes with $V_{2}$. The range of yield stress was from 700 dynes $/ \mathrm{cm}^{2}$ to 70,000 dynes $/ \mathrm{cm}^{2}(0.01 \mathrm{psi}$ to $1.0 \mathrm{psi})$. The range of viscosity was from 100 poise to 1000 poise, or $10,000 \mathrm{cP}$ to $100,000 \mathrm{cP}$. Ball velocity ranged from 0 to $1 \mathrm{~cm} / \mathrm{s}$. It is presently believed that the actual values for the tank wastes will be somewhere within these ranges, at least for the nonconvective region, and that the ball velocity will be approximately in the 0 to $2 \mathrm{~cm} / \mathrm{s}$ range in most cases. The conclusions from the error analysis presented here assume that the values for yield stress and viscosity are within the above stated bounds.

Table 1 shows some interesting features. Even for the smallest yield stress and the largest viscosity, the yield stress is the main contributor to the drag force. This is evidenced by the fact that for all cases the drag force does not increase in proportion to the velocity. It increases at a much smaller rate. When the yield stress is very high, a doubling of the velocity results in an increase in drag force of less than $1 \%$. It should already be clear that we will have difficulty making accurate viscosity determinations if the yield stress is very high. Except when the yield stress is very small the drag force is always in excess of $10^{6}$ dynes ( $10 \mathrm{~N}$ or $2 \mathrm{lb}$ ), so we should be able to make drag force measurements to within $1 \%$. with a $50 \mathrm{lb}$ load cell. Even for the case with very low yield stress, the drag forces are sufficiently high that $2 \%$ measurements of the drag force are possible with a $50 \mathrm{lb}$ load cell. This accuracy is probably acceptable.

Figures 9 and 10 show how the drag force varies over a selected range of viscosity and yield stress for two different velocities. All units in the plots are in cgs. Note the weak dependence on the viscosity and the strong dependence on the yield stress. The data in these figures are just a graphical representation of some of the data shown in Table 1.

Equation (29) is difficult to handle for purposes of analytic error analysis. Another expression relating drag force to yield stress and viscosity for Bingham fluids is given by Ansley and Smith (Eqs. (14) and (15)) as, 
Table 1. Calculated Values for Drag Forces $\left(T_{1}\right.$ and $\left.T_{2}\right)$ for Specific Values of Velocity $(V)$, Yield Stress $\left(\tau_{0}\right)$, Viscosity $\left(\mu_{0}\right)$, and

Ball Radius (R). All units are cgs.

\begin{tabular}{|c|c|c|c|c|c|c|}
\hline $\mathrm{R}$ & $V_{1}$ & $v_{2}$ & $\mu_{0}$ & $\tau_{0}$ & $T_{1}$ & $\mathrm{~T}_{2}$ \\
\hline 4.6 & 0.5 & 1 & $1.00 \mathrm{E}+02$ & $7.00 \mathrm{E}+02$ & $7.39 \mathrm{E}+05$ & $7.81 \mathrm{E}+05$ \\
\hline 4.6 & 0.5 & 1 & $1.00 \mathrm{E}+02$ & $3.50 \mathrm{E}+03$ & $3.42 \mathrm{E}+06$ & $3.51 \mathrm{E}+06$ \\
\hline 4.6 & 0.5 & 1 & $1.00 \mathrm{E}+02$ & $7.00 \mathrm{E}+03$ & $6.72 \mathrm{E}+06$ & $6.84 \mathrm{E}+06$ \\
\hline 4.6 & 0.5 & 1 & $1.00 \mathrm{E}+02$ & $3.50 \mathrm{E}+04$ & $3.28 \mathrm{E}+07$ & $3.30 \mathrm{E}+07$ \\
\hline 4.6 & 0.5 & 1 & $1.00 \mathrm{E}+02$ & $7.00 \mathrm{E}+04$ & $6.51 E+07$ & $6.55 \mathrm{E}+07$ \\
\hline 4.6 & 0.5 & 1 & $5.00 \mathrm{E}+02$ & $7.00 \mathrm{E}+02$ & $8.70 \mathrm{E}+05$ & $9.77 \mathrm{E}+05$ \\
\hline 4.6 & 0.5 & 1 & $5.00 \mathrm{E}+02$ & $3.50 \mathrm{E}+03$ & $3.69 \mathrm{E}+06$ & $3.91 \mathrm{E}+06$ \\
\hline 4.6 & 0.5 & 1 & $5.00 \mathrm{E}+02$ & $7.00 \mathrm{E}+03$ & $7.10 \mathrm{E}+06$ & $7.39 \mathrm{E}+06$ \\
\hline 4.6 & 0.5 & 1 & $5.00 \mathrm{E}+02$ & $3.50 \mathrm{E}+04$ & $3.36 \mathrm{E}+07$ & $3.42 \mathrm{E}+07$ \\
\hline 4.6 & 0.5 & 1 & $5.00 \mathrm{E}+02$ & $7.00 \mathrm{E}+04$ & $6.63 E+07$ & $6.72 \mathrm{E}+07$ \\
\hline 4.6 & 0.5 & 1 & $1.00 \mathrm{E}+03$ & $7.00 \mathrm{E}+02$ & $9.77 \mathrm{E}+05$ & $1.14 \mathrm{E}+06$ \\
\hline 4.6 & 0.5 & 1 & $1.00 \mathrm{E}+03$ & $3.50 \mathrm{E}+03$ & $3.91 \mathrm{E}+06$ & $4.22 \mathrm{E}+06$ \\
\hline 4.6 & 0.5 & 1 & $1.00 \mathrm{E}+03$ & $7.00 \mathrm{E}+03$ & $7.39 \mathrm{E}+06$ & $7.81 \mathrm{E}+06$ \\
\hline 4.6 & 0.5 & 1 & $1.00 \mathrm{E}+03$ & $3.50 \mathrm{E}+04$ & $3.42 \mathrm{E}+07$ & $3.51 E+07$ \\
\hline 4.6 & 0.5 & 1 & $1.00 \mathrm{E}+03$ & $7.00 \mathrm{E}+04$ & $6.72 \mathrm{E}+07$ & $6.84 \mathrm{E}+07$ \\
\hline 4.6 & 0.1 & 0.3 & $1.00 \mathrm{E}+02$ & $7.00 \mathrm{E}+02$ & $6.84 \mathrm{E}+05$ & $7.16 \mathrm{E}+05$ \\
\hline 4.6 & 0.1 & 0.3 & $1.00 \mathrm{E}+02$ & $3.50 \mathrm{E}+03$ & $3.30 \mathrm{E}+06$ & $7.37 \mathrm{E}+06$ \\
\hline 4.6 & 0.1 & 0.3 & $1.00 \mathrm{E}+02$ & $7.00 \mathrm{E}+03$ & $6.55 E+06$ & $6.65 \mathrm{E}+06$ \\
\hline 4.6 & 0.1 & 0.3 & $1.00 \mathrm{E}+02$ & $3.50 \mathrm{E}+04$ & $3.24 \mathrm{E}+07$ & $3.26 \mathrm{E}+07$ \\
\hline 4.6 & 0.1 & 0.3 & $1.00 \mathrm{E}+02$ & $7.00 \mathrm{E}+04$ & $6.46 \mathrm{E}+07$ & $6.49 \mathrm{E}+07$ \\
\hline 4.6 & 0.1 & 0.3 & $5.00 \mathrm{E}+02$ & $7.00 \mathrm{E}+02$ & $7.39 \mathrm{E}+05$ & $8.15 \mathrm{E}+05$ \\
\hline 4.6 & 0.1 & 0.3 & $5.00 \mathrm{E}+02$ & $3.50 \mathrm{E}+03$ & $3.42 \mathrm{E}+06$ & $3.58 \mathrm{E}+06$ \\
\hline 4.6 & 0.1 & 0.3 & $5.00 \mathrm{E}+02$ & $7.00 \mathrm{E}+03$ & $6.72 \mathrm{E}+06$ & $6.94 \mathrm{E}+06$ \\
\hline 4.6 & 0.1 & 0.3 & $5.00 \mathrm{E}+02$ & $3.50 \mathrm{E}+04$ & $3.28 \mathrm{E}+07$ & $3.33 \mathrm{E}+07$ \\
\hline 4.6 & 0.1 & 0.3 & $5.00 \mathrm{E}+02$ & $7.00 \mathrm{E}+04$ & $6.51 \mathrm{E}+07$ & $6.58 \mathrm{E}+07$ \\
\hline 4.6 & 0.1 & 0.3 & $1.00 \mathrm{E}+03$ & $7.00 \mathrm{E}+02$ & $7.81 E+05$ & $8.94 \mathrm{E}+05$ \\
\hline 4.6 & 0.1 & $0 . \dot{3}$ & $1.00 \mathrm{E}+03$ & $3.50 \mathrm{E}+03$ & $3.51 \mathrm{E}+06$ & $6.58 \mathrm{E}+07$ \\
\hline 4.6 & 0.1 & 0.3 & $1.00 \mathrm{E}+03$ & $7.00 \mathrm{E}+03$ & $6.84 E+06$ & $7.16 \mathrm{E}+06$ \\
\hline 4.6 & 0.1 & 0.3 & $1.00 \mathrm{E}+03$ & $3.50 \mathrm{E}+04$ & $3.30 \mathrm{E}+07$ & $3.37 \mathrm{E}+07$ \\
\hline 4.6 & 0.1 & 0.3 & $1.00 \mathrm{E}+03$ & $7.00 \mathrm{E}+04$ & $6.55 \mathrm{E}+07$ & $6.65 \mathrm{E}+07$ \\
\hline
\end{tabular}




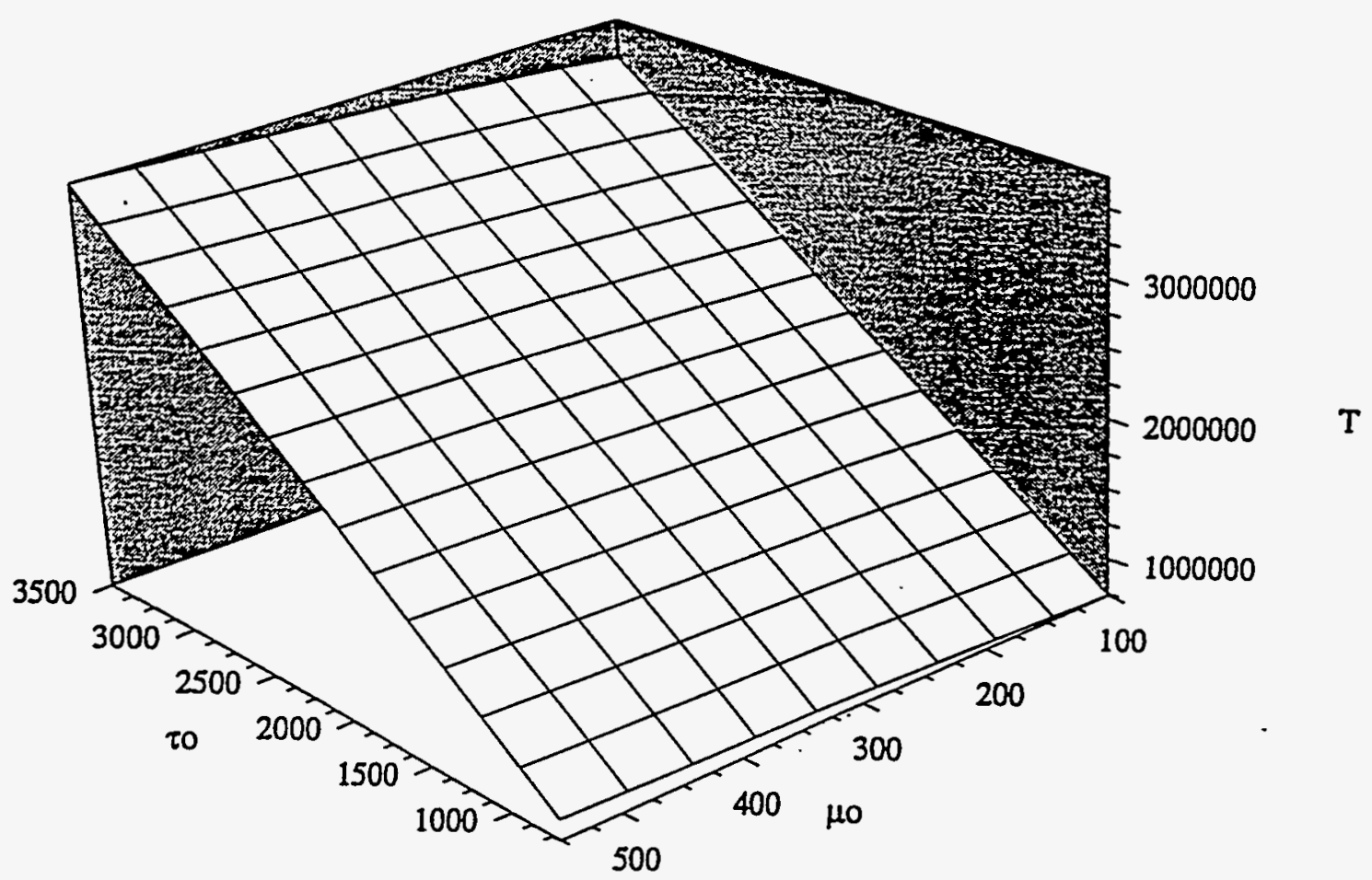

Figure 9. Calculated Drag Force $(T)$ as a Function of Yield Stress $\left(\tau_{0}\right)$ and Viscosity $\left(\mu_{0}\right)$ for a Ball Velocity of $0.5 \mathrm{~cm} / \mathrm{s}$. All units are in cgs.

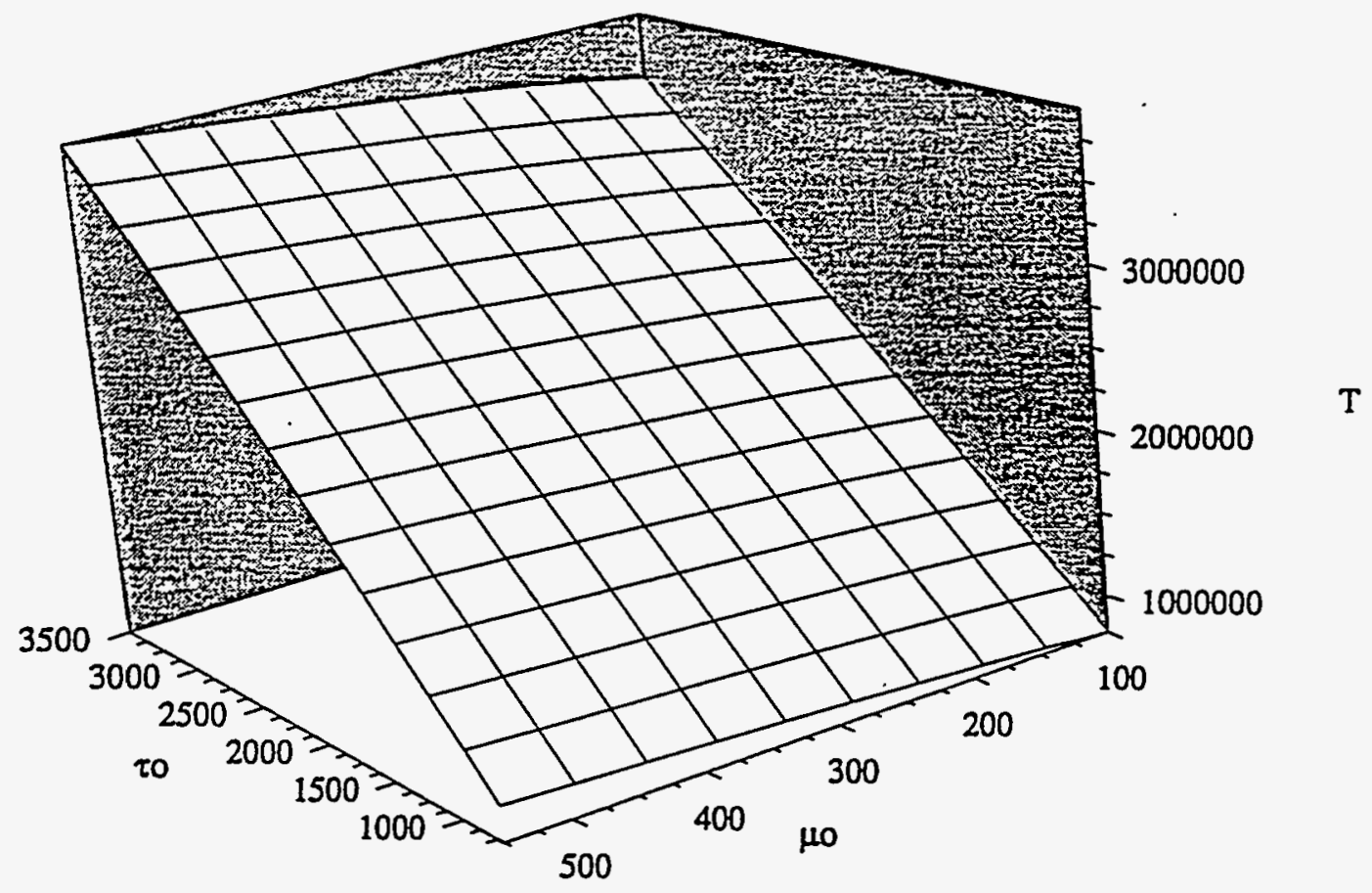

Figure 10. Calculated Drag Force $(T)$ as a Function of Yield Stress $\left(\tau_{\mathrm{a}}\right)$ and Viscosity $\left(\mu_{\mathrm{o}}\right)$ for a Ball Velocity of $1.0 \mathrm{~cm} / \mathrm{s}$. All units are in cgs. 


$$
T=6 \pi \mu_{o} R V\left[1+2 \frac{\tau_{o} R}{\mu_{o} V}\right]
$$

and 10 This equation gives results similar to those predicted by Beris et al. (1985). Substituting values for the ball radius and evaluating in cgs units, we obtain

$$
T=86 \mu_{0} V+788 \tau_{0}
$$

The drag force is the sum of two terms, the first of which involves only the viscosity and velocity and the second depends only on the yield stress and ball radius. For the parameter range of interest here, the second term will always be much larger than the first term. If we neglect the first term, the expression for the fractional error is

$$
\frac{\Delta \tau_{o}}{\tau_{o}}=\frac{\Delta T}{T}
$$

This expression shows that the fractional error in the determined yield strength is the same as the fractional error in the measured drag force. This result has also been verified in numerical calculations using Eqs. (29-32) where small errors were assigned to the measured drag forces. Systematic errors due to improper accounting of buoyancy forces and drag force on the cable will be the dominant contributors to errors in the yield stress. Measurement errors are negligible in comparison. However, as discussed above even systematic errors are not expected to be so large as to cause unacceptable errors in the determination of the yield stress.

In Eq. (34) it has been implicitly assumed that there is negligible error in the measurement of the ball radius. Since this radius can be precisely measured beforehand, this assumption is justified so long as the ball radius remains constant throughout its deployment in the tank. Crystal growth or accumulation on the ball during the course of experiments may result in an effective change in the dimension of the ball. For this reason, a polyethylene coating will be applied to the ball to reduce the possibilities of crystal growth. Tests performed in the laboratory using a chemical waste simulant at $45^{\circ} \mathrm{C}$ have shown that crystal growth does not occur on either a polyethylene-coated or bare tungsten carbide ball over time periods of one month. Therefore, we will neglect uncertainty in ball radius as a contributor to measurement errors.

The fluid viscosity is determined from measurements at two different velocities. Using Eq. (33) the resulting expression is,

$$
T_{2}-T_{1}=86 \mu_{o}\left(V_{2}-V_{1}\right)
$$


Note that systematic errors in the measured drag forces will not contribute to errors in the viscosity, since they will cancel in subtraction of the two forces. If we solve for $\mu_{0}$ in the above equation and use standard error analysis methods we find

$$
\frac{\Delta \mu_{0}}{\mu_{0}}=\frac{\Delta\left(T_{2}-T_{1}\right)}{T_{2}-T_{1}}+\frac{\Delta\left(V_{2}-V_{1}\right)}{V_{2}-V_{1}}
$$

The fractional error in the velocities is around $1 \%$ and the velocity differences are comparable to the velocities, so the error induced in the viscosity due to velocity measurement errors is of order $1 \%$ and is therefore entirely negligible. For the parameter range of interest, the differences in drag forces at two different velocities is small compared to the drag forces themselves (see Table 1). Therefore the uncertainty in the difference can be large even if each individual drag force can be measured to good precision. To good approximation then,

$$
\frac{\Delta \mu_{0}}{\mu_{0}}=\frac{\Delta\left(T_{2}-T_{1}\right)}{T_{2}-T_{1}}
$$

Using values shown in Table 1 for the drag forces at two different velocities $(0.5$ and $1.0 \mathrm{~cm} / \mathrm{s})$ and assuming a measurement error of $1 \%$ in one of the drag forces, we can calculate the expected error in $\mu_{\mathrm{o}}$ for a range of viscosities and yield strengths. The results are shown in Figure 11. The fractional error in viscosity increases with increasing yield strength and decreases with increasing viscosity. It appears that errors of the order of $10 \%$ to $200 \%$ can be expected in our determination of viscosity. This error can be reduced if the velocity range of the experiments is increased. In our calculations the upper limit on the velocity was $1 \mathrm{~cm} / \mathrm{s}$. From Eq. (35) it is seen that if the velocity difference is increased by some factor, then the error in viscosity will be reduced by the same factor. There may be some advantage in performing experiments at as high a velocity as possible consistent with remaining in the Stoke's regime. This is the reason why data collection at $2 \mathrm{~cm} / \mathrm{s}$ was suggested earlier in discussions about experimental procedure.

It should be noted that if the yield stress and viscosity are outside the bounds assumed in our calculations, then the resulting errors will also be different. For instance, if the viscosity becomes much lower than 100 poise, the resulting error will increase dramatically. This is because the viscous contribution to the drag force becomes very small.

Even if there were no yield stress at all, we would have difficulty measuring a drag force at a ball velocity of $1 \mathrm{~cm} / \mathrm{s}$ and a viscosity of 100 poise. The viscosity will have to be at least 100 poise if the ball rheometer is to provide reasonably accurate results. Since estimates of the viscosity are around 1000 poise, we expect that we will be able to perform tests in the NC layer. In the convective or mixed layers, the drag will simply be too small to measure accurately unless the ball velocity is increased. It is for this reason that ball velocities on the order of $1 \mathrm{~m} / \mathrm{s}$ are planned for this region.

The data will be used to determine a reasonable model for the fluid, with the present belief that a generalized Bingham fluid model may serve us quite well. However, if the viscosity and yield strength cannot be determined to sufficiently accurate precision, it may not be possible to choose 


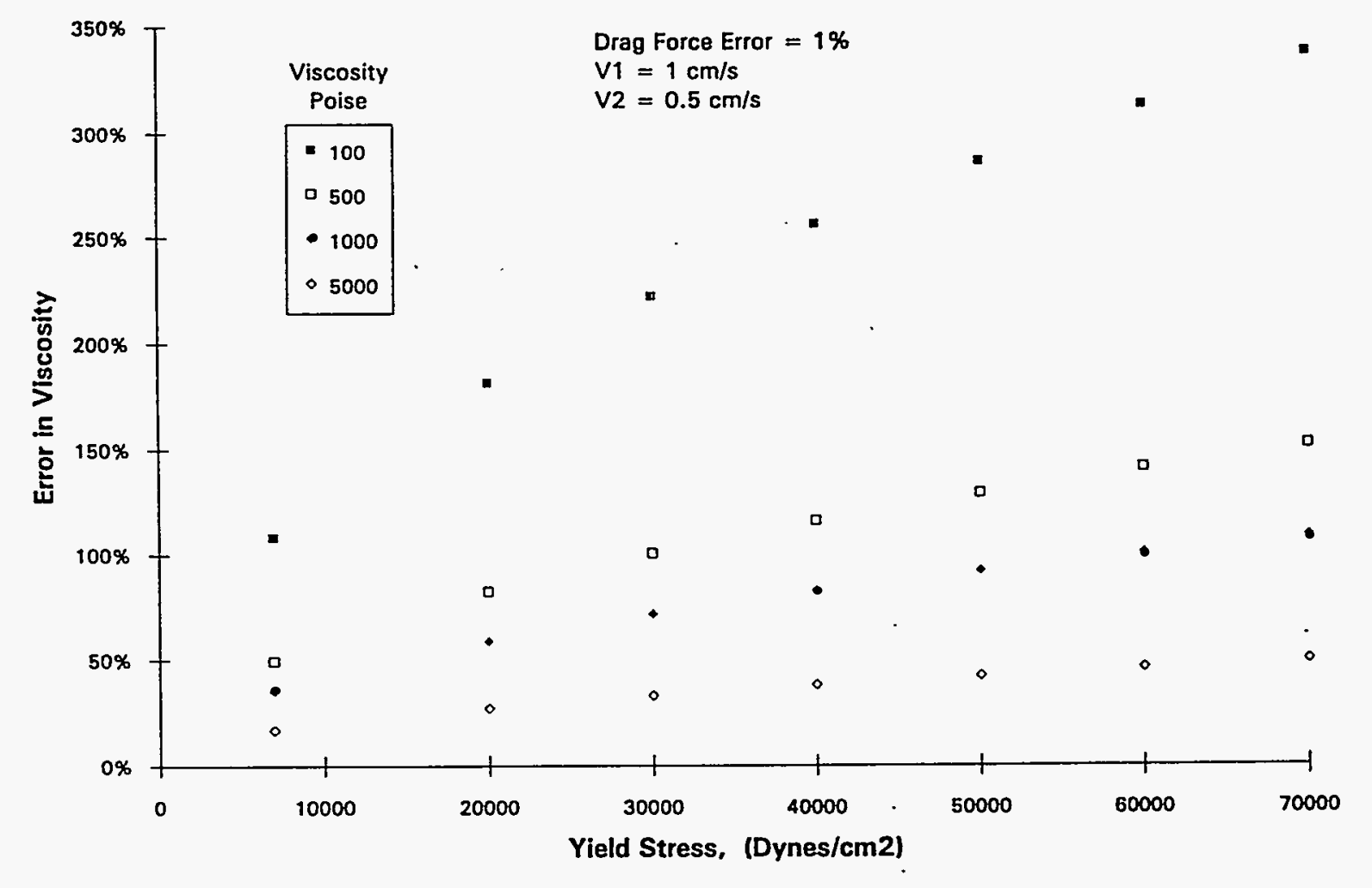

Figure 11. Fractional Error in Derived Viscosity for a Range of Viscosities and Yield Stresses. A Bingham plastic fluid model is assumed.

among several models based on the data. The behavior of the viscosity as a function of strain rate is the most important ingredient for any model. For instance, it may not be possible to choose between a Bingham fluid model and a generalized Bingham fluid model. On the other hand, it should be remembered that there is no strong requirement for precision modeling. A model which predicts the viscosity to about a factor of two is very acceptable to the currently identified data users community.

The sources for errors in the drag force have been described above. Some of them may be difficult to reduce. On the other hand, some of them will result in systematic errors which affect all measured drag forces in the same manner and these errors will not have a large effect on the derived viscosity. Systematic errors will include errors in the ball weight, the cable weight and drag force, the buoyancy force, and the pulley friction. Errors in load cell readings and errors due to waste inhomogeneity are examples of random errors. However, it is suspected that waste inhomogeneity will probably cause the largest confusion in interpretation and reduction of the data. Let us consider this factor.

The equations relating drag force and velocity to rheological parameters are steady-state equations. Therefore we must pull the ball until a steady-state condition is reached. On a plot of drag force vs. ball position, this will be evidenced by a relatively flat region in the plot. If fluid properties vary within the drag region, then the drag force will reflect these variations and it will be difficult to obtain a precise number for the drag force. Consider for example the data shown in Figures 12 and 13, obtained in the laboratory with a chemical simulant. Figure 12 indicates that a steady-state condition 


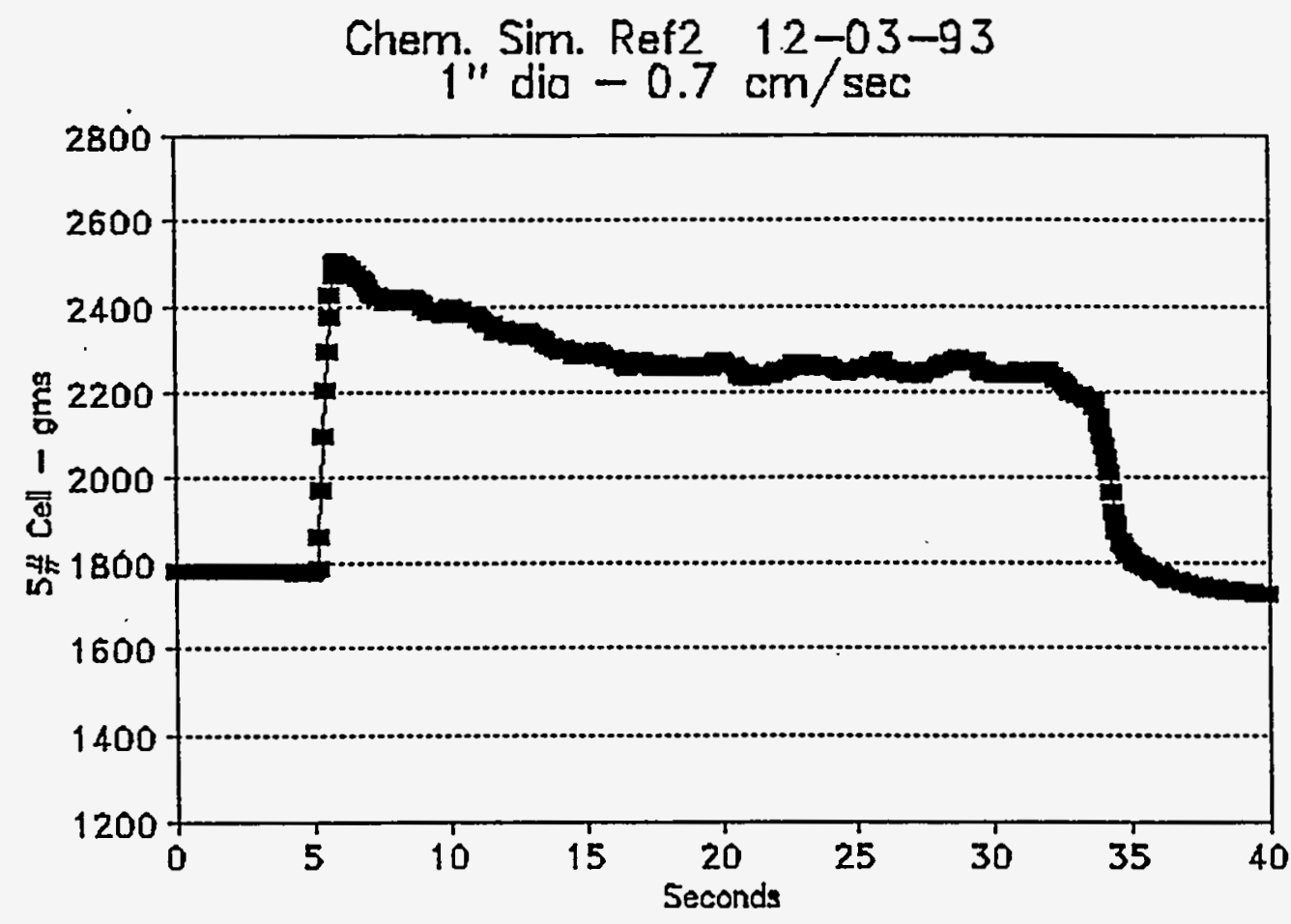

Figure 12. Drag Force Measured in a Chemical Simulant Using a 2.5-cm-Diameter Ball with a Velocity of $0.7 \mathrm{~cm} / \mathrm{s}$. A steady-state flow is apparently obtained.

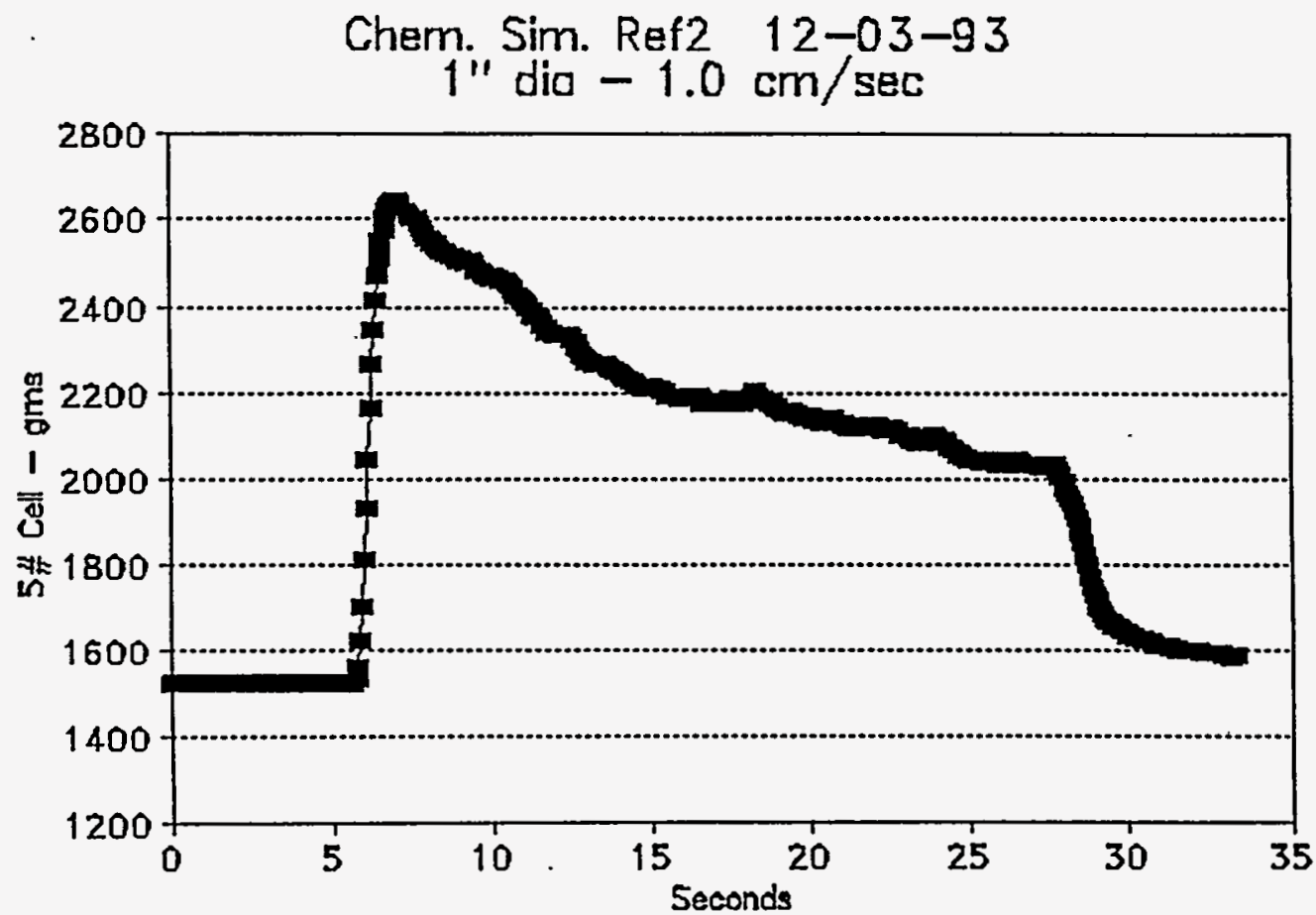

Figure 13. Drag Force Measured in a Chemical Simulant Using a 2.5-cm-Diameter Ball with a Velocity of $1.0 \mathrm{~cm} / \mathrm{s}$. No steady-state region is apparent. 
has been achieved and allows for a fairly precise determination of the drag force for the particular ball velocity employed. Figure 13, obtained with the same simulant, is quite different and it is clear that determination of the drag force to anything like $1 \%$ accuracy is not possible. In Figure 14 is shown a compendium of simulant data obtained over a range of velocities on two different days. If the Bingham plastic fluid model is applicable, then we would expect the drag forces to plot nearly linearly with velocity. The data at $1 \mathrm{~cm} / \mathrm{s}$ do not fit very well with the other points. It would be difficult to claim that we have determined the drag forces to good precision. Inhomogeneities in the fluid itself are believed to be the cause of the experimental scatter observed in the data. Tingey (1992) has measured yield stresses which vary in excess of $10 \%$ in window C core samples of the NC layer. Thus, even careful accounting of errors and application of the best instrumentation available may not be enough to guarantee highly accurate determination of fluid rheological properties. However, it is believed that by suitable analysis of the data and the development of averaging methods, we will be able to determine the yield stress and zero shear rate viscosity to acceptable accuracy.

In fact, we will be able to measure total cable tension quite accurately, and the discussion above is not about measurement errors but rather points out limitations concerning modeling, the subsequent measurement procedure, and the nature of the waste. The waste may not be structured in a manner which permits simple reduction in terms of some fluid model.

The error analysis performed to date has shown that errors in the drag force measurements are the most critical in determining how well we can derive rheological parameters. Velocity measurement

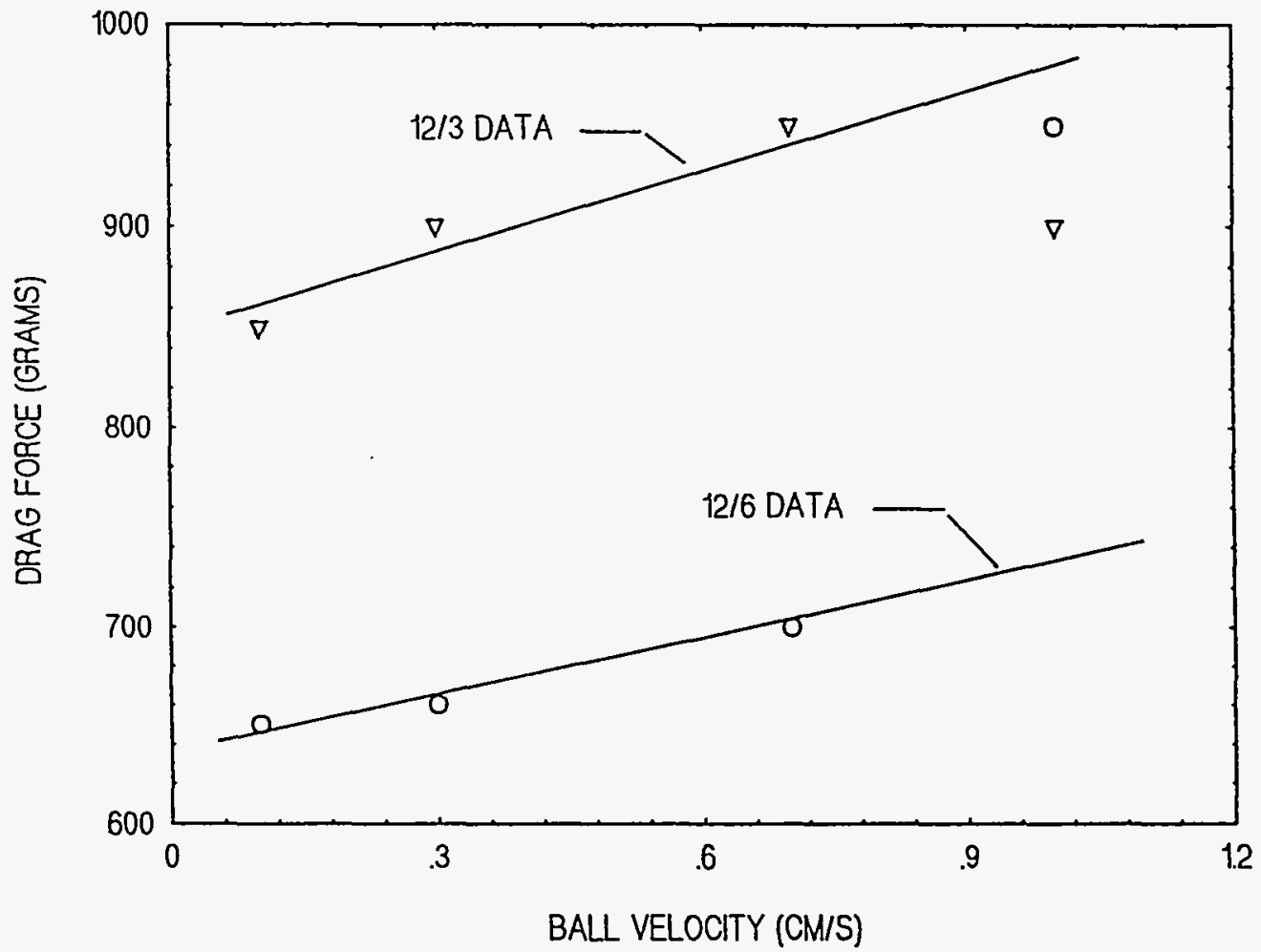

Figure 14. Drag Force Measured in the Same Chemical Simulant on Different Days 
error is expected to be unimportant. Errors in drag force measurement arise from two sources; compensation errors for forces which are not due to drag on the ball (such as cable and pulley weight, cable drag, pulley friction, and buoyancy) and errors due to accuracy limits on the load cells and the recording instruments. The compensation errors, which are systematic, will result mostly in errors in the derived values of the yield stress. Load cell measurement errors, which are random, will result in errors in the derived values for viscosity. The exact dependence of these errors on the drag force errors is dependent upon the model chosen to relate rheological parameters to the measured quantities. The analysis presented here assumed a Bingham plastic model. Other models can be expected to give somewhat different results, but qualitatively the results should be similar. Inhomogeneity of the fluid is expected to further add to the uncertainty in derived results.

\subsection{Additional Laboratory Measurements}

In the discussions presented above mention has been made of additional testing which will be required in order to prepare ourselves for the deployment of the ball rheometer and the subsequent analysis of the data. In this section we will briefly review these measurements.

\subsection{Tests to Establish Expected Ball Rheometer Behavior}

1. Test large ball in a simulant to determine expected pull distance needed to reach steady-state flow conditions. This test will require a fairly large volume of simulant in order to minimize boundary effects. A Bentonite clay mixture may be sufficient for this test. Tests should cover the range of Bingham numbers expected in actual waste. The effects of interfaces should also be examined in these experiments.

2. Perform tests to determine the expected drag on the cable. Repeat tests using only the ball and then the ball and cable. In this manner we can determine if the cable influences the drag force on the ball. Tests should be performed using a chemical simulant near tank temperature (about $50^{\circ} \mathrm{C}$ ). Present tests have been performed at room temperature.

3. Perform falling ball experiments in a chemical simulant. Test both constant force-variable velocity and constant velocity-variable force options. These tests may provide insight on which method is more promising in a waste which has substantial inhomogeneity.

4. Devise test to determine inter-related effects of buoyancy and waste yield strength on drag force measurements in a chemical simulant. Since the drag force scales as the square of the ball radius while the buoyancy scales as the cube of the radius, experiments with different size balls may provide clues. Simulants should include a Bentonite clay mixture as well as a chemical simulant.

5. Perform tests to determine the expected rheological properties of a well mixed chemical simulant. Tests thus far have focused on the NC layer. We need spindle rheometer data on a mixed waste, in addition to ball rheometer data. 


\subsection{Model Development Tests}

There is some belief that the initial overshoot in the drag force as the ball is being accelerated upwards may provide some rheological information, particularly about the fluid Bingham number. The existing theories on ball rheometry make predictions based on steady-state flow conditions. The overshoot is a transient phenomenon and has not been studied in detail. Limited studies with simulants in the laboratory have not shown the initial drag force spike to be a strong function of Bingham number, and little difference in the magnitude of the spike is observed over variations of a factor of ten in Bingham number. Theoretical investigation of this transient behavior should be conducted to determine the expected dependence of the start-up spike on rheological parameters. These predictions should then be tested in the laboratory using well characterized fluids as well as a chemical simulant.

Experimental tests will also be performed expressly for the purpose of developing a fluid model which describes the tank waste. These experiments will be performed using a chemical simulant of the waste.

We will perform a series of falling-ball tests using x-ray radiography. These tests will be performed with a simulated waste, which yields with an external force exerted on it. When real and simulated wastes are allowed to settle, they form the convective and nonconvective layers. In the convective layer, the density and solid fraction are less than in the nonconvective layer. The nonconvective layer shows a yield stress owing to the crystal formation. We will perform falling-ball tests for both uniformly mixed waste, which is yielded by mixing, and waste in the nonconvective layer, which is not yielded. We will use the waste simulant previously developed by LANL.

From falling-ball tests, we plan to learn more about the unexpected problems regarding in-situ rheology measurements. This information will give us guidelines for these measurements and their evaluation. We also will perform falling-ball tests using a 3.5-in. diameter tungsten ball, as in in-situ testing, using $x$-ray radiography. Los Alamos will develop computer software for the data reduction for in-situ measurements. Below, we briefly detail these tests.

\subsubsection{Waste Simulant Characterization Tests}

The objective of these tests is to characterize the simulant in terms of densities and solid fraction as a function of a given initial recipe, temperature, and time.

\subsubsection{Falling-Ball Tests with Yielded Waste Simulant}

The objective of these tests is to measure the viscometric behavior of the uniformly mixed waste simulant with the falling-ball technique, using x-ray radiography as a function of temperature and solid fraction. A necessary objective is to verify that the yielded waste simulant can be characterized as a continuum.

\subsubsection{Falling-Ball Tests with Unyielded Waste Simulant}

The objective of these tests is to measure the viscometric behavior of the unmixed waste simulant after it has had sufficient time to stratify. The falling-ball technique, using $x$-ray radiography, will be 
used to probe the simulant as a function of temperature and solid fraction, assuming that the simulant can be characterized as a continuum.

\subsubsection{Viscosity Measurements with Other Methods, Using the Yielded Waste Simulant}

We intend to perform additional viscosity measurements, using different techniques to compare the falling-ball measurements with other techniques.

\subsubsection{Flow Visualization in Falling-Ball Tests to Determine the Shape of Yielded Region}

The purpose of this test is to determine the shape of the yielded envelope around a falling ball in the unyielded waste simulant experimentally. This information will verify the prediction of the choice of constitutive equation and, if needed, the numerical model.

\subsubsection{Falling-Ball Tests with 3.6-in. Diameter Tungsten Ball}

The objective of these tests is to obtain data for unyielded waste uising a 3.6-in. diameter tungsten ball and $\mathrm{x}$-ray radiography at representative conditions. Similar experiments will be conducted in a test setup in which the ball is pulled with a constant velocity.

\subsection{Conclusions}

Knowledge of the rheological properties of the waste material contained in Hanford Site high-level radioactive waste tanks is crucial for the success of many of the mitigation/remediation schemes. The ball rheometer is being developed as a means for obtaining in-situ rheological information about the waste. Initial deployment of this device will be in Tank 101-SY. In this report we have discussed the need for the ball rheometer and have described in some detail its mechanical structure, its mode of operation, and the factors which will influence its utility in the measurement of the waste's rheological properties.

Current rheology data has been shown to have significant uncertainty. For example, there is a considerable difference in the $C$ layer viscosity for samples from windows $C$ and $E$. Rheology measurements on core samples from NC layer have shown anomalous results. Typically, the rheograms show that the shear stress is a decreasing function of the strain rate at low strain rates. This behavior is not observed as a quasi-steady and stable theogram for any other material (and/or slurries) investigated in the literature. NC layer data also indicate that over a certain range of temperatures and strain rates, viscosity increases with increasing temperature, contrary to the behavior of most fluids.

The experimental method (cone-and-plate viscometer) previously used in obtaining the rheology data from the core samples also has a number of drawbacks. One of the most important is that the clearance of the cone-and-plate viscometer is on the same order as the crystalline sizes existing in the waste (the crystalline particles in the waste slurry are believed to be 1 to $100 \mu \mathrm{m}$ in size). For a cone-and-plate viscometer used in the hot-cell measurements, this particle size is likely to interfere with the gap, resulting in erroneous viscosity or yield stress measurements. 
In order to test the adequacy of cone and plate for the waste rheology measurements, a series of laboratory tests were performed using different viscometers and a chemical simulant of the waste. These tests showed that the cone-and-plate viscometer yields reasonably repeatable results for Newtonian fluids and non-Newtonian fluids with very fine particles. The measured values for these fluids using the cone-and-plate viscometer were also in good agreement with the values obtained using cylindrical spindles. The cone-and-plate viscometer gave significantly different readings from spindles for the simulated waste. The repeatability of a test for the cone and plate was also unacceptable and showed unacceptable scatter. Spindles, however, gave reasonably repeatable results for the same waste simulant. We concluded that with the crystalline sizes on the same order as the clearance of the cone and plate, the cone-and-plate viscometer will provide unacceptable measurements and therefore this rheometer is not recommended for the rheology measurement of the wastes. Although spindles gave repeatable results, the measured quantity was in question because of the concern associated with the particle migration in a shear flow field. Additional tests with a falling ball also were performed. These preliminary tests showed shear thinning behavior. However, the measured values were similar to those obtained using the spindle method.

The waste's yield stress was measured previously by the vane method and the method of measuring the shear stress at which flow first begins under constant shear stress or shear rate conditions. There are significant differences (about $100 \%$ ) between values obtained from the vane method and the method of measuring the shear stress at the onset of flow under constant shear rate. When the values obtained from the two tests mentioned above are compared with the value obtained from an indirect method, they differ by orders of magnitude.

The need for better in-situ measurements of the rheological properties of the waste is therefore established. The advent of mitigation techniques such as the mixer pump have also created a need for instrumentation with which the effectiveness of a method can be measured. The ball rheometer can provide data from which the effectiveness of the mixer pump can be determined at least to some degree. Simple location of the boundary separating the convective or mixed layer from the nonconvective layer will allow a determination of the influence of the mixer pump on the waste.

We have pointed out some limitations on the capability of the ball rheometer. It is most likely to provide rheological information about the NC layer. The $\mathrm{C}$ or mixed layer may have a yield stress and viscosity which are too low to measure accurately, and it is possible that only upper bounds will be established. It is expected to provide a better measurement of density in the mixed or convective layer than in the nonconvective layer. Interfaces between fluid layers may introduce difficulties in the reduction of data. Stratified or inhomogeneous material in the nonconvective layer are also likely to present problems in the reduction of data.

Rheological properties of the waste can be measured in-situ with the ball rheometer. The yield stress and viscosity can be obtained from the measured drag force and ball velocity using a correlation for the drag coefficient. This approach will give reasonable results if the fluid behavior is identified correctly. For a generalized Bingham plastic, at least three tests with different ball velocities at the same elevation are necessary to evaluate the rheology of the waste from the measured drag force and velocity. If the solid and gas concentrations do not vary significantly over a distance of several feet (i.e., if the rheology is constant), then acceptably accurate determinations of yield stress and viscosity are possible. The ball rheometer will provide the most accurate results if the waste viscosity and/or the yield stress are appreciably high. Therefore it is expected to be most applicable to the NC layer. For low viscosities (less than $1000 \mathrm{cP}$ ), such as may exist in the convective or a mixed layer, and 
near zero yield stresses, the drag force on the ball becomes very small and is not a strong function of the ball velocity.

An analysis of errors and the manner in which they manifest themselves in the derived rheological values has been performed. This analysis shows that systematic errors are most important for the determination of the yield stress while even small random errors in the measurement of the drag forces can have a large effect on the determination of the viscosity. Choice of the most suitable model to describe the fluid behavior may be difficult based solely on the data. However, the magnitude of expected errors presently appears acceptable to users of the data, and there may be no strong need to determine the most appropriate model.

Additional testing in the laboratory with simulants is necessary before the ball theometer can be expected to provide reliable information from in-situ measurements in the tank. Testing to determine the effects of ball and cable interaction and drag on the cable as well as the effective buoyancy in the fluid must be performed. In addition, the pull distance necessary to arrive at a steady-state condition needs to be determined. Several other tests have been described. Laboratory testing with chemical simulants is also necessary for development of a model which we believe will adequately describe the waste fluid and with which we can reduce the ball rheometer data. Development of a reasonably accurate fluid model will be very important in contributing to the success of the ball rheometer.

The ball rheometer will be successful if it can provide the following information:

1. density in the convective or mixed region,

2. location of the boundary between the convective and nonconvective region,

3. yield stress and viscosity of the nonconvective region to within $50 \%$ of true values, and

4. upper bound to the apparent viscosity of the convective or mixed region.

Accomplishment of the above may be difficult and depends somewhat on the nature of the waste material, which is not known to sufficient detail at present. For instance, if the yield stress of the NC layer is too high, it may be necessary to manually assist the ball to the bottom of the tank. If inhomogeneities are too great within the NC layer, we may have difficulty in reducing the drag force data. If the fluid nature of the waste cannot be captured in some fluid model, then reduction of the ball rheometer data may not be accomplished to within the required precision. The resolution of some of these issues will not be accomplished until actual in-situ data are acquired. However, it is presently felt that these issues can be dealt with and that the ball rheometer will accomplish the measurement goals for which it has been designed.

\subsection{References}

Abbott, J. R., H. Brenner, A. L. Graham, and L. A. Mondy. 1991a. "Techniques for Analyzing the Behavior of Concentrated Suspensions," Liquid-Solid Flows-1991. American Society of Mechanical Engineers, New York, New York, FED-Vol. 118, pp. 1-11. 
Abbott, J. R., N. Tetlow, A. L. Graham, S. A. Altobelli, E. Fukushima, L. A. Mondy, and T. S. Stephans. 1991b. "Experimental Observations of Particle Migration in Concentrated Suspensions: Couette Flow," J. Rheology 35(5).

Adachi, K. and N. Yoshioka. 1973. "On Creeping Flow of a Visco-Plastic Fluid Past a Circular Cylinder," Chem. Eng. Scie. 28, 215-226.

Ansley, R. W. and T. N. Smith. 1967. "Motion of Spherical Particles in a Bingham Plastic," AIChE $J$ 13, 1193-1196.

Beris, A. N., J. A. Tsamopoulos, R. C. Armstrong, and R. A. Brown. 1985. "Creeping Motion of a Sphere through a Bingham Plastic," J. Fluid Mech 158, 219-244.

Bhavaraju, S. M., R. A. Mashelkar, and H. W. Blanch. 1978. "Bubble Motion and Mass Transfer in Non-Newtonian Fluids," AIChE Journal 24 (6), 1063-1070.

Brookes, G. F. and R. L. Whitmore. 1969. "Drag Forces in Bingham Plastics," Rheologica Acta 8 (4), pp. 472-482.

Brookes, G. F. and R. L. Whitmore. 1968. "The Static Drag on Bodies in Bingham Plastics," Rheologica Acta 7(2), 188-193.

Chhabra, R. P. 1993. Bubbles, Drops, and Particles in Non-Newtonian Fluids, CRC Press, Boca Raton, Florida, Ch. 3.

Chhabra, R. P., C. Tiu, and P. H. T. Uhlherr. 1981. "Creeping Motion of Spheres through Ellis Model Fluids," Rheologica Acta 20, 346.

Chhabra, R. P. 1983. "Some Remarks on 'Drag Coefficients of Slowly Moving Sphere in NonNewtonian Fluids'," J. Non-Newtonian Fluid Mechanics 13, 225-227.

Dazhi, G. and R. I. Tanner. 1985. "The Drag on a Sphere in a Power Law Fluid," J. NonNewtonian Fluid Mechanics 17, 1-12.

Dedegil, M. Y. 1987. "Drag Coefficient and Settling Velocity of Particles in Non-Newtonian Suspensions," ASME Journal of Fluids Engineering, Vol. 109, 319-323.

Dzuy, N. and D. V. Boger. 1983. "Yield Stress Measurement for Concentrated Suspensions," Journal of Rheology 27(4), 321-349.

Dzuy, N. and D. V. Boger. 1985. "Direct Yield Stress Measurement with the Vane Method," Journal of Rheology 29(3), 335-347.

Ferguson, J. and Z. Kemblowski. 1991. Applied Fluid Rheology, Elsevier Science Publishing Company, Inc., New York, New York. 
Gerhart, P. M., and R. J. Gross. 1985. Fundamentals of Fluid Mechanics, Addison-Wesley Publishing Company, Redding, Massachusetts.

Goodrich, K., A. Yoshimura, and R. K. Prud'Homme. 1989. "Measurement of the Modulus and Yield Strength of Soft Gels: Experiments and Numerical Simulation," Journal of Rheology 33(2), 317-327.

Ito, S. and T. Kajiuchi. 1969. "Drag Force on a Sphere Moving in Plastic Fluid," Journal of Chemical Engineering of Japan 2(1), 19-23.

Keentok, M., J. F. Milthorpe, and E. O’Donovan. 1985. "On the Shearing Zone around Rotating Vanes in Plastic Liquids: Theory and Experiment," Journal of Non-Newtonian Fluid Mechanics 17, 23-35.

Merrington, A. and L. W. Woodcock. 1991. "Rheometric Experiments on Idealized Suspensions: Phase Behavior and Scaling Laws," Liquid-Solid Flows-1991, American Society of Mechanical Engineers, New York, New York, FED-Vol. 118, 109-115.

Mondy, L. A., A. L. Graham, and J. L. Jensen. 1986. "Continuum Approximations and Particle Interactions in Concentrated Suspensions," J. Rheology 30(5), 1031-1051.

Navickis, L. L. and E. B. Bagley. 1983. "Yield Stresses in Concentrated Dispersions of Closely Packed, Deformable Gel Particles," Journal of Rheology 27(6), 519-536.

Pasamehmetoglu, K.O., C. Unal, and J.N. Edwards. 1993. The Evaluation of Methods for Rheology Measurements of the Waste Contained in Hanford Tank 241-SY-101, LA-UR-93-4300, N-6-93-R152. Los Alamos National Laboratory, Albuquerque, New Mexico.

Piccola Jr., J. P., E. C. Sams, and R. A. Tennant. 1993. Rheological Parameters for Simulants of Hanford Tank SY-101, LA-UR-93-2600. Los Alamos National Laboratory, Albuquerque, New Mexico.

Powell R. L., and L. A. Walla. 1991. "Viscosity Measurement of Suspensions of Rod Mixtures," Liquid-Solid Flows-1991, American Society of Mechanical Engineers, New York, New York, FEDVol. 118, 117-122.

Shepard, C. L. 1994. Report on Data Requirements and Hardware Selection for In-Situ Ball Rheometer, PNL-10171. Pacific Northwest Laboratory, Richland, Washington.

Tran, Q. K., D. T. Trinh, R. R. Horsley, and J. A. Reizes. 1993. "Drag Coefficients and Settling Velocities of Spheres in Yield: Pseudoplastic Slurries," ASME Winter Meeting, AMD-Vol. 175, Developments in Non-Newtonian Flows, 131-137.

Yoshioka, N. and K. Adachi. 1971. "On Variational Principles for a Non-Newtonian Fluid," Journal of Chemical Engineering of Japan 4(3), 13-16. 
Zhu, J. and M. G. Satish. 1992. "Non-Newtonian Effects on the Drag of Creeping Flow through Packed Beds," Int. J. Multiphase Flow 18, 765-767. 


\section{Distribution}

No. of

Copies

QFFSITE

12 DOE Office of Scientific and Technical Information

Billy C. Hudson

202 Northridge $C t$.

Lindsborg, KS 67456

J. L. Kovach

Nuclear Consulting Services, Inc.

P.O. Box 29151

Columbus, OH 43229-0151

Thomas E. Larson

Los Alamos National Laboratory MS-P915, DX-DO

P.O. Box 1663

Los Alamos, NM 87545

4 Los Alamos National Laboratory

P.O. Box 1663

Los Alamos, NM 87545

Attn: J. Abbott, K555

K. Pasamehmetoglu, K555

C. Unal, K555

J. R. White, K555

D. Pepson

U.S. Department of Energy

Trevion II Building, EM-35

Washington, D.C. 20585-0002

Dana Powers

Sandia National Laboratory

Dept. 6404/MS-0744

P.O. Box 5800

Albuquerque, NM 87815-0744
No. of

Copies

QNSITE

4 DOE Richland Operations Office

R. F. Christensen

S7-54

R. E. Gerton

S7-54

J. M. Gray

S7-54

G. W. Rosenwald

S7-54

24 Westinghouse Hanford Company

W. T. Alumkal

S7-85

H. Babad

S7-30

R. E. Bauer

L6-37

G. T. Bear

R1-51

T. R. Benegas

N1-27

L. E. Efferding

$\mathrm{H} 0-33$

R. L. Fritz

B4-08

C. E. Hanson

H5-09

G. D. Johnson

S7-15

N. W. Kirch

R2-11

L. S. Krogsrud

R3-08

D. C. Larsen

R1-51

J. W. Lentsch (5)

S7-15

R. M. Marusich

H4-65

N. G. McDuffie

S7-15

M. A. Payne

S7-14

D. A. Reynolds

R2-11

C. P. Shaw

L5-07

T. I. Stokes

H5-09

J. E. Van Beek 
No of

Copies

31 Pacific Northwest Laboratory

J. M. Al-heimer

K5-22

W. J. AI ' $\mathrm{y}$

S7-71

Z. I. Antoi.iak

K7-15

R. M. Bean

P8-08

S. Q. Bennett

K7-90

J. B. Colson

K5-10

E. J Eschbach

K7-15

J. D. Hudson

K7-15

B. M. Johnson

K1-78

F. E. Panisko (5)

P8-34

K. P. Recknagle

K7-15

H. C. Reid

K7-15

P. A. Scott

P7-43

C. L. Shepard

K5-25

C. W. Stewart (5)

K7-15

T. I. Stokes

H5-09

D. S. Trent

K7-15

Publishing Coordination

Technical Report Files (5) 